\title{
Digitisation of Traditional Craft Processes
}

XENOPHON ZABULIS*, Foundation for Research and Technology (FORTH), Institute of Computer Science (ICS), Greece

CARLO MEGHINI ${ }^{\dagger \dagger}$, Consiglio Nazionale delle Ricerche (CNR), Istituto di Scienza e Tecnologie della Informazione (ISTI), Italy

ARNAUD DUBOIS $\stackrel{+\ddagger}{\ddagger}$, Conservatoire National des Arts et Métiers (CNAM), Histoire des Technosciences en Société (HT2S), France

PARASKEVI DOULGERAKI*, NIKOLAOS PARTARAKIS*, ILIA ADAMI*, EFFIE KARUZAKI*, and ANNE-LAURE CARRE ${ }^{\S}$, Conservatoire National des Arts et Métiers (CNAM), Musée des Arts et Métiers, France

NIKOLAOS PATSIOURAS* and DANAE KAPLANIDI I, Piraeus Bank Group Cultural Foundation (PIOP), Greece

DANIELE METILLI ${ }^{\dagger}$ and VALENTINA BARTALESI ${ }^{\dagger}$, Consiglio Nazionale delle Ricerche (CNR), Istituto di Scienza e Tecnologie della Informazione (ISTI) CHRIS RINGASI and ELEANA TASIOPOULOU I, Piraeus Bank Group Cultural Foundation (PIOP) ZINOVIA STEFANIDI*, Foundation for Research and Technology (FORTH), Institute of Computer Science (ICS)

\footnotetext{
The Mingei project has received funding from the European Union's Horizon 2020 Innovation Action under Grant Agreement No. 822336 (http://www.mingei-project.eu). MoCap recordings and segmentation results were acquired by the Association pour la Recherche et le Developpement des Methodes et Processus Industriels (ARMINES) in the context of its contribution in the Mingei Innovation Action. The authors thank the Association of Friends of Haus der Seidenkultur and the Chios Mastiha Growers Associations for the demonstrations and the provision of insights in understanding the crafts of silk textile manufacturing and mastic cultivation, respectively. The authors thank Centre de Recherche Européen de Recherche et de Formation aux Arts Verriers (CERFAV) for providing a workshop for the glasswork and Jean-Paul Mateus and Dominique Jamis for their participation in the glasswork demonstrations and ethnography.

Authors' addresses: X. Zabulis, Foundation for Research and Technology (FORTH), Institute of Computer Science (ICS), N. Plastira 100, Vassilika Vouton, Heraklion, Crete GR-700 13, Greece; email: zabulis@ics.forth.gr; C. Meghini, Consiglio Nazionale delle Ricerche (CNR), Istituto di Scienza e Tecnologie della Informazione (ISTI), Area della Ricerca CNR, via G. Moruzzi 1, Pisa 56124, Italy; email: carlo.meghini@isti.cnr.it; A. Dubois, Conservatoire National des Arts et Métiers (CNAM), Histoire des technosciences en Société (HT2S), Case 1LAB10, 2 rue Conté, Paris 75003, France; email: arnaud64.dubois@gmail.com; P. Doulgeraki, N. Partarakis, I. Adami, E. Karuzaki, and A.-L. Carre, Conservatoire National des Arts et Métiers (CNAM), Musée des Arts et Métiers, Case 3MAM01, 292 rue Saint-Martin, Paris Cedex 03, 75141 France; emails: \{vdoulger, partarak, iadami, karuzaki\}@ics.forth.gr, anne-laure.carre@lecnam.net; N. Patsiouras and D. Kaplanidi, Piraeus Bank Group Cultural Foundation (PIOP), 6 Ang. Gerontas St., Athens 105 58, Greece; emails: patsiouras@ics.forth.gr, danae.kaplanidi@gmail.com; D. Metilli and V. Bartalesi Consiglio Nazionale delle Ricerche (CNR), Istituto di Scienza e Tecnologie della Informazione (ISTI), Area della Ricerca CNR, via G. Moruzzi 1, Pisa, Italy, 56124; emails: \{daniele.metilli, valentina.bartalesi\}@isti.cnr.it; C. Ringas and E. Tasiopoulou, Piraeus Bank Group Cultural Foundation (PIOP), 6 Ang. Gerontas St., Athens, Greece, 105 58; emails: \{riggasch, tasiopouloue\}@piraeusbank.gr; Z. Stefanidi, Foundation for Research and Technology (FORTH), Institute of Computer Science (ICS), N. Plastira 100, Vassilika Vouton, Heraklion, Crete, Greece, GR-700 13; email: zina.stef@gmail.com.

Permission to make digital or hard copies of all or part of this work for personal or classroom use is granted without fee provided that copies are not made or distributed for profit or commercial advantage and that copies bear this notice and the full citation on the first page. Copyrights for components of this work owned by others than ACM must be honored. Abstracting with credit is permitted. To copy otherwise, or republish, to post on servers or to redistribute to lists, requires prior specific permission and/or a fee. Request permissions from permissions@acm.org.

(c) 2022 Association for Computing Machinery.

1556-4673/2022/09-ART53 $\$ 15.00$

https://doi.org/10.1145/3494675
} 
An approach to the representation and documentation of craft processes is proposed. The proposed approach is a method for the systematic identification and digital representation of pertinent data, information, and knowledge. The outcome representation is compatible with contemporary digitisation practices and digital preservation standards. The implementation of the approach is provided within the context of an online platform that is accompanied by auxiliary tools for digital curation. This platform is a multiple user system, where craft representations can be collaboratively authored, shared, displayed, and digitally preserved in standardised formats. Basic uses of this scheme and presentational applications are provided, along with identification of future work and limitations.

\section{CCS Concepts: • Information systems $\rightarrow$ Digital libraries and archives;}

Additional Key Words and Phrases: Cultural Heritage, traditional crafts, digital preservation, Semantic Web, process modelling

\section{ACM Reference format:}

Xenophon Zabulis, Carlo Meghini, Arnaud Dubois, Paraskevi Doulgeraki, Nikolaos Partarakis, Ilia Adami, Effie Karuzaki, Anne-Laure Carre, Nikolaos Patsiouras, Danae Kaplanidi, Daniele Metilli, Valentina Bartalesi, Chris Ringas, Eleana Tasiopoulou, and Zinovia Stefanidi. 2022. Digitisation of Traditional Craft Processes. J. Comput. Cult. Herit. 15, 3, Article 53 (September 2022), 24 pages.

https://doi.org/10.1145/3494675

\section{INTRODUCTION}

Crafts, handicrafts, traditional, or "heritage crafts" [44] are an integral part of Cultural Heritage [51], which is in many cases endangered due to the "declining number of practitioners and apprentices" [103]. Thus, their preservation means the continuation of their practice [47]. Therefore, accurate representations, documentation, instructions, and training aids support the preservation of craft knowledge and practice.

Authoritative reports on crafts and conservation [13,24] identify tangible and intangible craft dimensions. Tangible dimensions regard materials, tools, and workspaces. Intangible dimensions refer to know-how and skill, but also collective memories, values, and traditions. We refer to contextual and crafting intangible dimensions. Contextual dimensions refer to the social and historical context and their representation is studied separately in [69]. Crafting dimensions refer to the role of the mind in handicraft activities.

The pursued crafting process representation spans heterogeneous dimensions and, thus, multiple types of "digitisation" are required. Appearance and geometrical properties of physical phenomena are recorded, or "digitised," in "signal" representations, i.e., audio-visual, 3D/4D. Meaning and interpretation are encoded using knowledge statements in "semantic" representations. We use the term "digitisation" in the ampler sense that considers the digital representation, or digitisation, of a craft as composed of both signal and semantic content. This digitisation is implemented by a semantic model that associates the recording of actions in a 1-1 correspondence with their interpretations. The model is Semantic Web compliant to enable digital preservation of both digital recordings and their interpretations. The implementation is offered as part of the Mingei Online Platform (MOP) [69], a Web-based, collaborative, authoring environment. The MOP facilitates management, interlinking, and curation of digital assets and contextualisation knowledge, stemming from the study of a craft. In this work, the MOP is extended with UI components that accommodate the instantiation of the introduced classes, in Section 3, for the representation of crafting dimensions.

The remainder of this work is illustrated in Figure 1 and is structured as follows. In Section 2, efforts in the documentation and representation of crafts and crafting processes are overviewed. Prior work employed in this work is cited in Section 2.3. The proposed approach is an authoring method, or protocol, that leads to the representation of crafting processes, in six phases. These phases are outlined at the start of Section 3 and elaborated in six subsequent and respective subsections. In Section 4, the implementation of each phase is presented, in an equal number of subsections. In Section 5, applications are found in the digital preservation, documentation, and presentation of crafting processes. In Section 6, a summary of this work is provided along with an identification of its limits and directions for future work. 


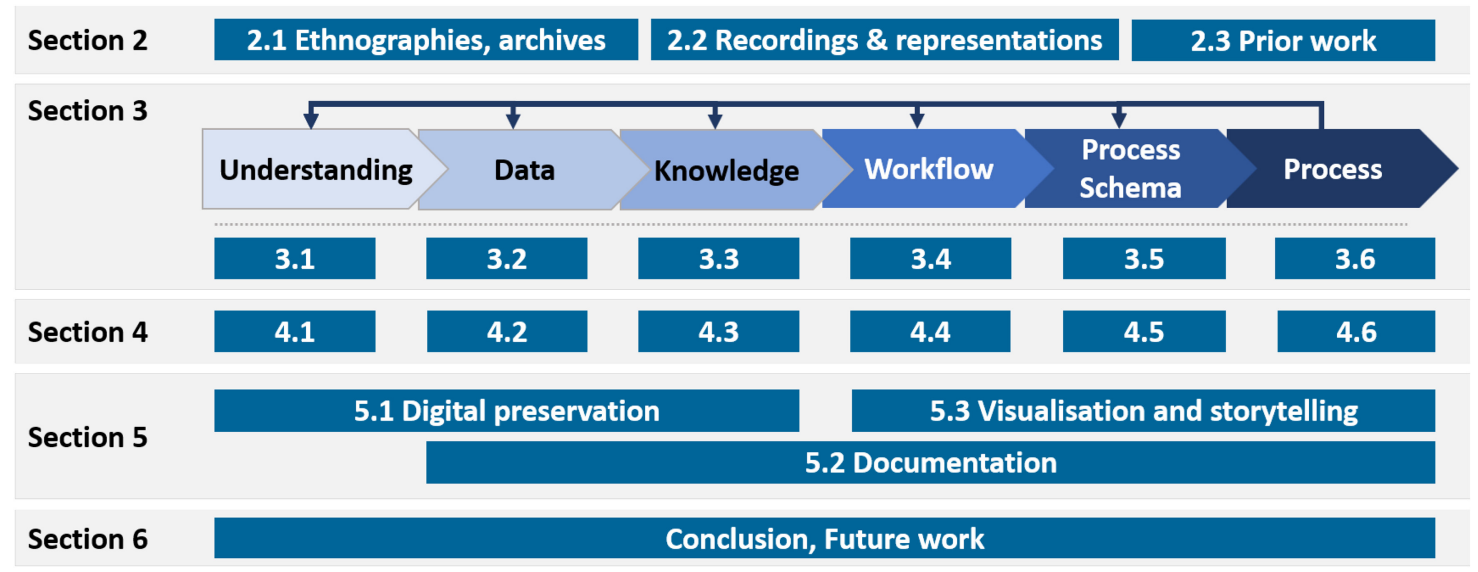

Fig. 1. Structure of this work.

Abbreviations. $\mathrm{CH}$, Cultural Heritage; $\mathrm{CIDOC}$, International Committee for Documentation (Comité International pour la documentation); CIDOC-CRM, CIDOC Conceptual Reference Model [21]; EDM, Europeana Data Model, [23]; FRBRoo, Functional Requirements for Bibliographic Records-object oriented; HTML, Hypertext Markup Language; HTTP, Hypertext Transfer Protocol; IMU, Inertial Measurement Unit; ICCROM, International Centre for the Study of the Preservation and Restoration of Cultural Property; ICOM, International Council of Museums; ICH, Intangible Cultural Heritage; IPR, Intellectual Property Rights; IRI, Internationalised Resource Identifier; MOP, Mingei Online Platform; MR, Mixed Reality; MoCap, Motion Capture; OWL, W3C Web Ontology Language; $\mathbb{R}^{3}$, the set of real numbers; $R D F$, Resource Description Framework [15]; $S O(3)$, the 3D rotation group; SPARQL, Protocol and RDF Query Language; UI, User Interface; UML, Unified Modelling Language; URL, Uniform Resource Locator; UNESCO, United Nations Educational, Scientific and Cultural Organization; VR, Virtual Reality; W3C, World Wide Web Consortium; WWW/Web, World Wide Web; XML, Extensible Markup Language.

All Uniform Resource Locators (URLs) were accessed on the submission date. In the electronic version, images are highly magnifiable.

\section{BACKGROUND}

\subsection{Preservation of Crafts as Cultural Heritage}

United Nations Educational, Scientific and Cultural Organization's (UNESCO's) recognition of traditional crafts as Intangible Cultural Heritage (ICH) [103] and support in the creation of national inventories [104], generated awareness and compliance of craft descriptions according to scientific and ethical guidelines. Descriptions can be found in the Representative List of the ICH of Humanity of UNESCO [102] and national inventories, e.g., [6, 63, 64]. The descriptions emphasise cultural content, social components, ownership, Intellectual Property Rights (IPR), and authenticity certification, but do not include sufficient technical descriptions to support craft reenaction.

In 1990, UNESCO published a data collection guide for the documentation of traditional crafts [45]. Though technologically outdated, it identifies the essential elements to be recorded: artefacts, materials, tools, and the crafting process. Questionnaires and forms are provided to systematise data collection. Photographic documentation is deemed necessary to record the practicalities of the crafting process, such as the way to hold a tool and manipulate it. Cinematography and digital technologies facilitated the recording of directly comprehensible, visual examples. Video dictionaries of crafting gestures were proposed in [109]. In [50], a worn camera was proposed to capture the viewpoint of the practitioner. The 3D recording of crafting motion was used to enable any viewpoint in [20]. 
Ethnography [57] identifies and describes the activity of a social unit as "textual construction of reality" [3]. Recently, it has been applied in the workshop, with examples in carpentry [108], glasswork [4], and textile manufacturing [49].

\subsection{Information and Communication Technologies}

Digital resources are systematically used in the documentation of Cultural Heritage $(\mathbf{C H})$. Developed methods identify Signal representations, which are audio-visual recordings and other measurements of the physical world and its events. Semantic representations organise information about the recorded entities, to create interlinked knowledge entities, in such a way that meaning can be deducted.

2.2.1 Tangible Heritage. In the signal domain, digitisation targeted the faithful representation of material and visual properties. The principal methods for capturing the structure and appearance are photography and 3D scanning. Historically, the MINERVA programme [16] paved the way for the establishment of good practices for digitisation in 2D images and standardisation of imaging and video formats. More recently, the 3D ICONS project has provided an analogous contribution for 3D digitisation [14]. In the semantic domain, efforts were made to contextualise digital assets. This was achieved by linking semantic metadata to digital assets relevant to the creator, the date of creation, the location of the artefact, the history of conservation events, and so on. Pertinent approaches relied on the tradition in library and archival science, creating object-centric or collection-centric descriptions (MINERVA [16], Europeana Rhine [8], etc.). These efforts enabled the formal representation of data, metadata, and knowledge, as well as the development of online repositories of semantically linked content.

2.2.2 Intangible Heritage. In the signal domain, digitisation enabled the recording of performances in multiple media and formats, exhibiting immersive qualities and novel interactive experiences [72]. For verbal content, pattern recognition is increasingly utilised in the transcription of manuscripts [46, 94], oral tradition, theatrical and vocal performances [98], and similarly for musical content [7]. MoCap and Computer Vision are used to capture articulated human motion in 3D, documenting body motion in dance $[20,26,100]$ and theatre [65]. In the semantic domain, multi-disciplinary projects fostered collaboration between information scientists and $\mathrm{CH}$ professionals. In [9], sharing of knowledge was supported, by assigning meaning to text. In [40], heterogeneous collections were linked under common concepts [40]. Exploration of collections through conceptual links was explored in [39]. In [17], collections were presented as narratives. In [87], ways to create interactive stories for cultural sites were developed. In [62], preservation methods for cultural and scientific resources were proposed. In the more recent of these works, the focus shifted on event-centric representations, in response to the drawbacks and scarce utility of object-centric representations.

2.2.3 Vocational Training. ICTs contribute through $2 \mathrm{D} / 3 \mathrm{D}$ annotation and simulation in vocational training. In [33], the need for visual annotation upon photographic documentation in handicrafts was identified. Mixed Reality (MR) and Virtual Reality (VR) environments were used for training professionals in manual tasks [66]. In [12], human motion is simulated, for workspace design. In [67], avatars are used for training in collaborative manual tasks. In [10], VR is employed for training in maintenance tasks. In [25], immersive storytelling is proposed for training.

\subsection{Prior Work}

The Mingei Craft Ontology [60] has been developed for the representation of crafts and harmonizes the following ontologies: (a) Narrative Ontology [59], extending the Conceptual Reference Model (CRM) with narratological concepts, (b) Functional Requirements for Bibliographic Records-object oriented (FRBRoo), which represents narration structure [22], and (c) World Wide Web Consortium (W3C) Web Ontology Language (OWL) Time ontology to represent temporal knowledge [42]. The implementation of the ontology is based on Semantic Web standards: (a) Resource Description Framework (RDF) as the basic data model for knowledge; 
(b) OWL 2 DL [54] for axiomatising the represented knowledge; (c) SPARQL [41] as the query language. The specification of the ontology can be found at [61].

The MOP is an authoring platform for the semantic representation of cultural, social, and historic knowledge on $\mathrm{CH}$, in general, and traditional crafts, in particular. The MOP implements the CRM and contains the Mingei ontology classes for objects, collections, sites, places, persons, and multimedia objects. In this work, the MOP is extended to implement the representation of classes introduced in Section 3.

\section{THE PROPOSED APPROACH}

Artefact creation is considered a process that is based on an "archetypa" plan, or a schema. This process schema, is conceptual and ostensive, in that it can be demonstrated and verbally described, i.e., as instructions. Audiovisual recordings phenomenologically capture the activity, while semantic descriptions enable the interpretation and abstraction of this activity as a stepwise process. The undetermined nature of handcrafting individual craft articles, or "the workmanship of risk" [88], is reflected by process schemas that support branching points to represent practitioner decisions taken during practice, or "on the fly." The proposed representation analytically associates the segments of these recordings with process steps, to support the abstraction of process schemas from the study of process recordings.

As a top-level ontology, CRM provides "very general concepts like space, time, matter, object, event, action, and so on, which are independent of a particular problem or domain" [38]. These classes and properties are overly generic to describe the specific concepts of crafting processes. To represent "concepts depending both on a particular domain and task," we have extended CRM classes, creating CRM subclasses and sub-properties to preserve interoperability [38]. The details of the classes introduced in this section can be found in Appendix A.

The proposed approach is a method for the identification and the digitisation of the signal and semantic dimensions of crafting processes, in the form of a six-phase protocol that leads to the implementation of the proposed representation. These phases are outlined below and illustrated in Figure 1. The illustration shows the promotion of knowledge from observation and understanding to formal representation. The feedback arrows refer to the revisit of phases to enhance or refine the representation.

(1) Craft understanding targets the identification of the workflow of the crafting processes, the location of practice, the involved objects, and its actors. The output is human-comprehensible documentation encoding the acquired knowledge.

(2) Data collection is the recording of physical objects and human actions leading to the transformation of materials into articles of craft, using visual, 3D, and motion sensors.

(3) Knowledge representation refers to the definition of fundamental types of semantic metadata, or basic knowledge elements, from which the representation will be comprised. These are the places, materials, tools, and roles encompassed by the crafting process.

(4) Workflow representation refers to the univocal representation of the process in steps and interrelating conditions between them, using activity diagrams.

(5) Process schema representation refers to the transcription of this activity diagram into a formal representation that prescribes the crafting process.

(6) Process representation refers to the annotation and linking of craft activity recordings to process steps.

To formulate the proposed approach, we use the following definitions.

A tool is an object or body member employed to make use of an affordance it bears [36], e.g., scissors provide the affordance of cutting. A machine is an apparatus comprised of Archimedean Simple Machines. An action is an event that consists of doing something intentionally by some agent of action. An activity is a set of actions carried out by one or more persons. Thus, activities are events. The entities involved in the crafting process are either endurants or perdurants, corresponding to "continuants" and "occurrents" [2] in Basic Formal Ontology, 

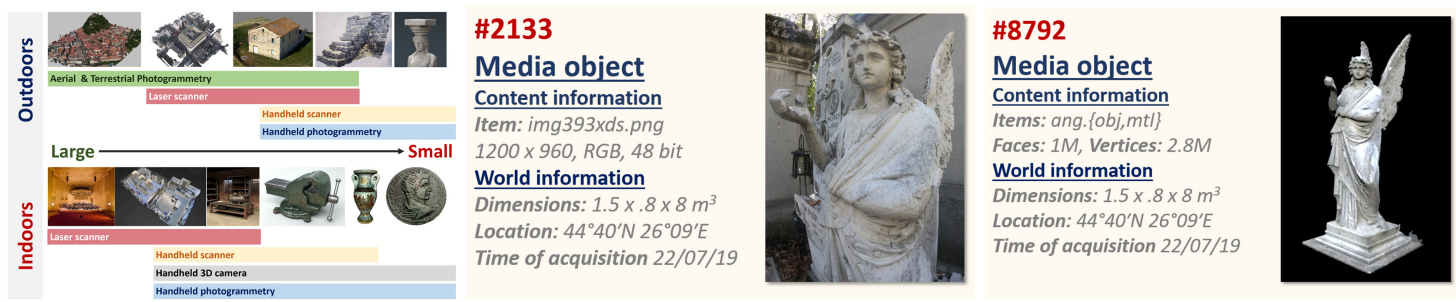

Fig. 2. Applicability of digitisation modalities (left) and illustration of media objects (middle, right) for endurant entities.

respectively. Endurants are materials, tools, machines, workplaces, and craft products. Perdurants are actions and natural phenomena.

A crafting process is a set of activities that transform materials into articles of craft. A workflow is an orchestrated and repeatable activity, enabled by the organisation of resources into processes. A crafting process schema is a representative prescription for how a set of activities should operate in a workflow to regularly achieve desired outcomes [97]. To refer to parts of a process schema or process, we say that they are comprised of step schemas and steps, respectively, independently of their depth in the process schema or process.

\subsection{Craft Understanding}

An ethnographic approach to the understanding of the crafting process is to consider it from a problem-solving viewpoint, as in [55, 92]. From this perspective, the abstraction of a process schema can be treated similarly to an algorithm. This treatment is convenient as the crafting process schema may have decision points and, thus, alternate workflows. Decision points are relevant to properties of individual pieces of material and environmental conditions, as well as practitioner observations and judgements. Besides decision points, process steps may refer to parallel or combined activities, by one or more persons. Some steps take place only to handle exceptional events, such as a repair for a mistake or an accident.

This phase has two outputs. The first is a vocabulary of terms with verbal definitions and visual descriptions of the involved knowledge entities. The second is a thick description [34] that enables the study of the activity beyond the context of a visual demonstration. This is implemented in the form of a storyboard, which serves as a detailed explanation of the crafting process that is visually captured and encodes temporal order, spatial arrangements, and purpose of actions. We call storyboards sequences of images or brief videos, in chronological order, enhanced with text and audiovisual annotations.

\subsection{Data Collection}

The endurant and perdurant components of the crafting process are digitally recorded. We use the term media object to abstract the heterogeneity of pertinent media types. The organisation of the recording sessions is facilitated by the vocabulary and storyboard, in identifying the objects, sites, and practitioner actions to be digitised. The obtained digital assets are assigned with technical metadata and unique identifiers.

Endurants are objects and sites and recorded through photographs and textured 3D meshes. The digital assets are images and textured 3D meshes. The choice of scanning modality for the 3D capture of endurants depends on subject size, material, and type of environment. In Figure 2 (left) an applicability guide is outlined. Also, in Figure 2, the media objects for a photograph (middle) and a 3D reconstruction [29] (right) are illustrated.

Perdurants are practitioner postures and gestures. They are recorded through audio, video, and motion sensing. Motion data are acquired by motion sensors and video. They are time-series of 3D locations, each one recording the 3D motion of a point on the surface of the practitioner's body. The measurements are topologically organised in a skeletal tree, a hierarchical data structure that represents avatar joints and limbs and is rooted at the avatar's 


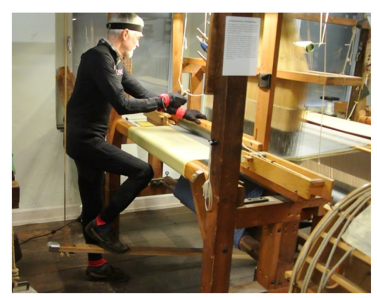

\#8792

Media object Motion Capture Meta-data Item: anim 3124.bvh Frame rate: $60 \mathrm{fps}$ Joints model, Limb sizes

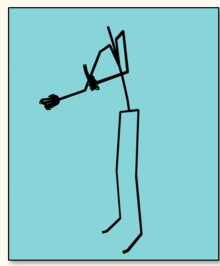

\#6759

Media object

Visual tracking

Meta-data

Item: image 1223.png

Resolution: $1200 \times 960$

Depth 24 bits per pixel

Frame rate $30 \mathrm{fps}$

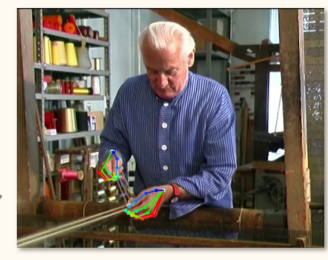

Fig. 3. Illustrations of media objects for perdurant entities.

torso. Branches of this tree are called body members. A posture is the configuration of the skeletal tree at a moment in time. A gesture is a chronologically ordered sequence of postures. The pose, i.e., location and orientation, of a held object is represented relative to a designated body member and is encoded as a rigid transformation, i.e., 3D rotation and translation. In Figure 3 (right), the media objects for two perdurants are illustrated. The left image shows a moment in a Motion Capture (MoCap) recording session and the middle image illustrates the obtained digital asset. The right image illustrates a media object obtained from markerless motion estimation in an archive documentary [19], its preview superimposing the estimated skeletal tree upon the original footage. The animations can be found in [85] (middle) and [82] (right).

Process recording may include alternate expressions of the schema, recorded by repeating steps of the schema that did not occur in the initial performance. Unforeseen decision points are noted and used to revise the vocabulary and the storyboard.

\subsection{Knowledge Representation}

The endurant and perdurant entities of the crafting process are represented. The representation is modelled with a few classes, called basic knowledge elements. Their instances contain semantic metadata and links to digital assets.

A basic knowledge element is an instance of any of the following CRM classes or their extensions. E5 Event for events, E57 Material for materials, and E53 Place for locations. It is noted that E39 Actor is a super class of E21 Person or E74 Group. Tool and Product extend E22 Man-Made Object. From [59], we adopt class ActorWithRole (Appendix A, Table 1), which extends E39 Actor and is used to describe the role of a pratictioner in an event. We also adopt MObject from [59] for the representation of media objects, which extends E73 Information Object. Instances of the above classes are used in declarative statements that link them to semantic metadata or media objects.

In Figure 4, knowledge elements and some of the relations between them are illustrated, using arrows. On the left, shown is an object-centric example, about a sculpture of "Tinian marble craftsmanship" [106]. An instance of Product is linked with its digitisation, an MObject. Its creation is an E5 Event, linked to a location (E53 Place) and a sculptor (ActorWithRole). The creation event is linked with the sculpture representation, by a causality link (red arrow), implemented as P15 was influenced by (influenced). On the right, is an event-centric example, about a process step (E5 Event) that took place during the creation of a glass carafe. This step, an E5 Event, was performed by the glassworker, using a blowpipe to insufflate air within a gather of liquid glass and create a glass bubble. The process step has a causality relation, of type P15 was influenced by (influenced) (red arrow), to the formation of the glass bubble (Product). This process step is linked to a process schema step, called "bubbling." Linked Mobject instances are the 3D digitisation of the blowpipe and two independent recordings of the practitioner motion, using MoCap and video analysis.

\subsection{Workflow Representation}

To unambiguously encode craft understanding, activity diagrams are proposed. An activity diagram is a flowchart that models workflows. Activity diagrams are borrowed from the Unified Modelling Language (UML) [37], 

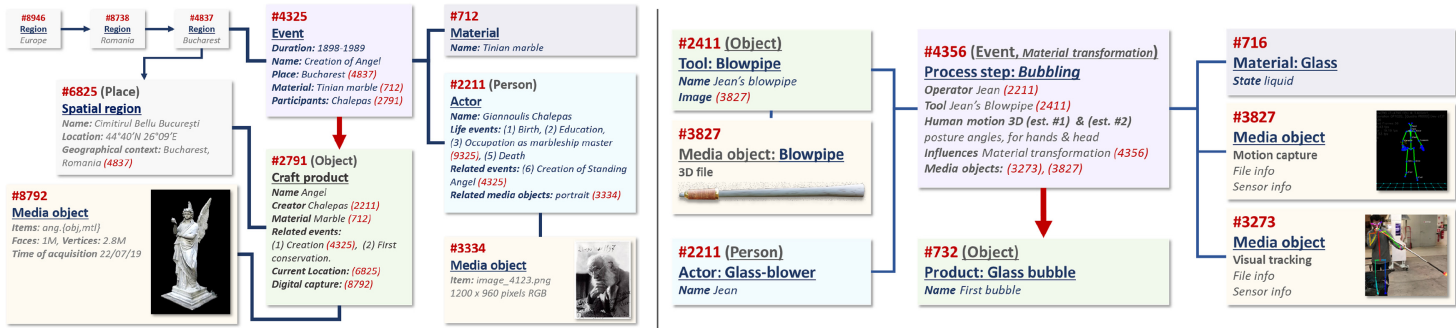

Fig. 4. Illustrations of knowledge elements and the relations between them.

but used with a slightly different meaning. In the UML, the nodes of the activity diagram represent computational actions that transform data. In this work, they represent physical actions that transform materials. Consequently, the transition types Transition, Fork, Merge, Foin, and Branch are adopted and denoted as in UML.

By recursive analysis, activity diagrams can be refined in a coarse-to-fine fashion. This hierarchical analysis is a modularization mechanism that is based on inclusion relations between steps. It allows a process schema to be seen as a single step in a larger, encompassing process schema.

\subsection{Process Schema Representation}

The activity diagram is transcribed into a transition graph. Classes Process schema and Process schema step are introduced (Appendix A, Table 2), to model process schemas and their steps, respectively, both extending E29 Design or Procedure. This way, property hasSubSteps enables the linkage of arbitrarily deep decompositions, as hasSubStep is a sub-property of P69 has association with, which generalises relations between instances of E29 Design or Procedure.

Transitions between steps are modelled using the following classes (Appendix A, Table 3).

- Transition represents an unconditional passage from one step to the next.

- Fork connects a schema step with subsequent schema steps performed in parallel and has a single input and multiple outputs.

- Merge is a node where two or more control paths unite. It has two or more input flows and one output flow.

- foin connects a schema step with the schema step that should be completed before any transition to the next step, and with the next step to be performed. It is implemented through nodes with multiple input steps and a single output step. They are structurally identical to merge nodes, except that the semantics are different: a join is synchronisation across a set of parallel flows, while in a merge only a single flow is active.

- Branch connects a step with a decision step that accepts tokens on one incoming edge and selects one outgoing alternative. Branch nodes control the flow of a process by selecting one of several alternatives, based on the outcome of a condition evaluation. To model decision steps, we introduce class Alternative that models the alternative paths stemming from a decision step.

Forks, joins, and merges may have more than one input or output state. Branches require the association of additional information. If transitions were modelled as relations between steps, then properties with arity greater than 2 would be required; but such are not admitted in semantic modelling, in general, and Semantic Web languages, in particular. Consequently, transitions are reified by introducing the above classes, each one capturing one transition type and encompassing a corresponding set of properties.

\subsection{Process Representation}

Process representations account for events that took place during a process. The representations of events include links to the knowledge entities that contextualise them. Classes Process and Process step are introduced 
(Appendix A, Table 4) to model processes and their steps, respectively. Both classes extend E7 Activity. Linking property hasProcessStep enables arbitrarily deep decomposition analysis, as hasProcessStep is a sub-property of $P 9$ consists of, which associates an instance of E4 Period with another instance of E4 Period, in the respective space-time region.

A Process is one of the multiple alternative outcomes of a Process schema. Intuitively, a process is an individual flow of events, out of those possible in the activity diagram. A process may or may not conform to a process schema, as explained in Section 4.6.

\section{IMPLEMENTATION}

The implementation is demonstrated in the authoring of crafting process representations.

\subsection{Craft Understanding}

Craft understanding follows ethnographic principles and includes background research of secondary sources. We refer to [110] for the identification of basic topics for craft understanding. In addition to ethnography, interviews with practitioners are invaluable in the understanding of craft practice, as they interpret the recorded events, identify skills, and recommend ways of teaching or presentation. The encoding of processes is reviewed by the practitioner(s), producing the final representation after some iterations. Background research before the ethnography increases its efficacy, saving time from the comprehension of basic vocabulary and notions.

\subsection{Data Collection}

Data collection regards the recording of static structures and events. These recordings are abstracted as media objects when inserted in the knowledge base.

Digitisation of endurants employs several scanning modalities, each operational in a specific range of spatial scales and environment types, as outlined in Figure 2 (left). Rooms and outdoor areas, require the combination of laser scanning and aerial photogrammetry to systematically cover. For smaller artefacts, photogrammetric reconstruction and active illumination sensors are nowadays simple and widely accessible. In Figure 5 (top), dry-stone walling structures [107] were digitised, registering interior laser scans with aerial and terrestrial photogrammetry scans, to provide wide coverage and detailed views. The dataset can be found at [28] and a video overview at [86]. We distinguish between the potential historic significance of objects and their utility in the crafting context. In the latter, modelling their geometrical structure can be sufficient. Albeit less realistic, the use of synthetic models simplifies digitisation and reduces scanning costs. The collection of tools for glasswork and "mastic cultivation" [105] used in this work is synthetic and was developed in [48].

The way that tools and materials are manipulated is captured in video and motion recordings. The applicability of MoCap and video modalities depends on the type of environment. Inertial MoCap [96] is more suitable than optical [31] in the cluttered space of workshops, due to reduced installation requirements and independence to occlusions. Nevertheless, inertial MoCap is not sufficiently sensitive to minute motions. To capture finger posture and motion, mechanical MoCap gloves are more suitable and less obtrusive, as many are "fingerless." Marker-less methods exhibit the least accuracy [11, 95], but require only a camera and are the only way to treat archive video. We found markerless motion recording suitable in obtaining key hand postures and body gestures and used [89] and [68] for these tasks, respectively. In Figure 5 (bottom), the left image is a photograph from the MoCap recording of a glassworker. The middle image is a rendering of the recorded 3D posture at that moment. The right image shows the result of marker-less motion estimation, rendered as a stick figure from two viewpoints.

New digital assets that are not Web resources, i.e., new media objects, are made such by uploading them to the MOP repository, associating them with new URLs, and assigning them to the Web server of the MOP. They are identified by an Internationalised Resource Identifier (IRI) and served by the MOP Web server via 


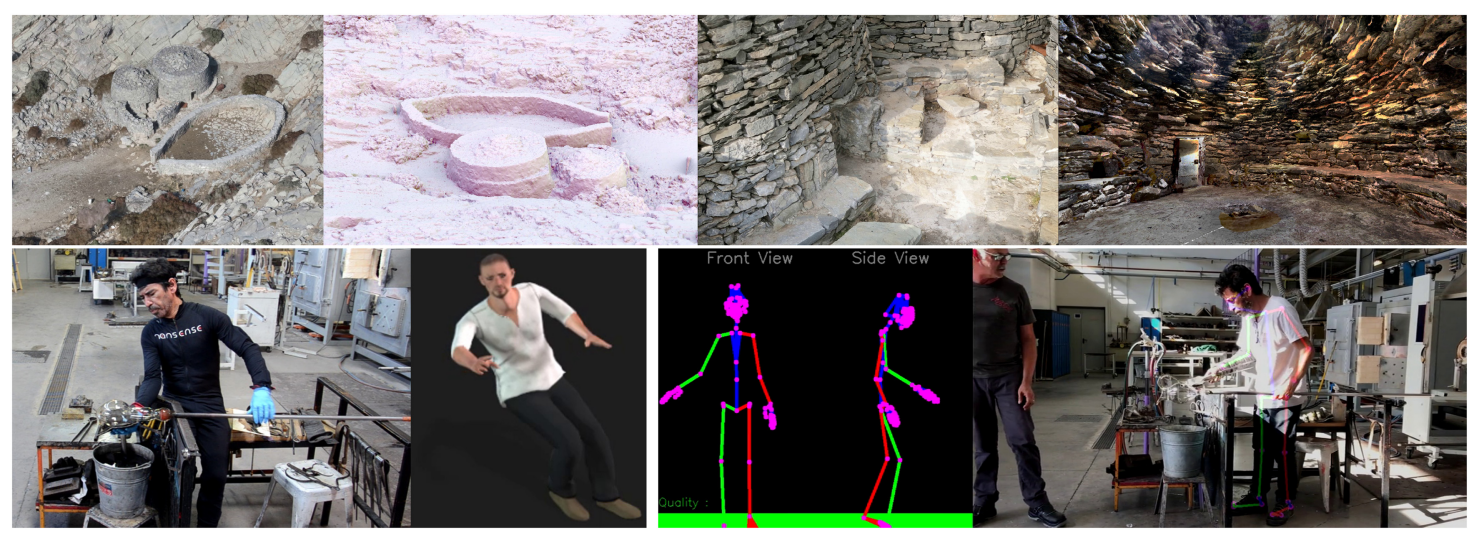

Fig. 5. Digitisation of endurant (top) and perdurant (bottom) entities.

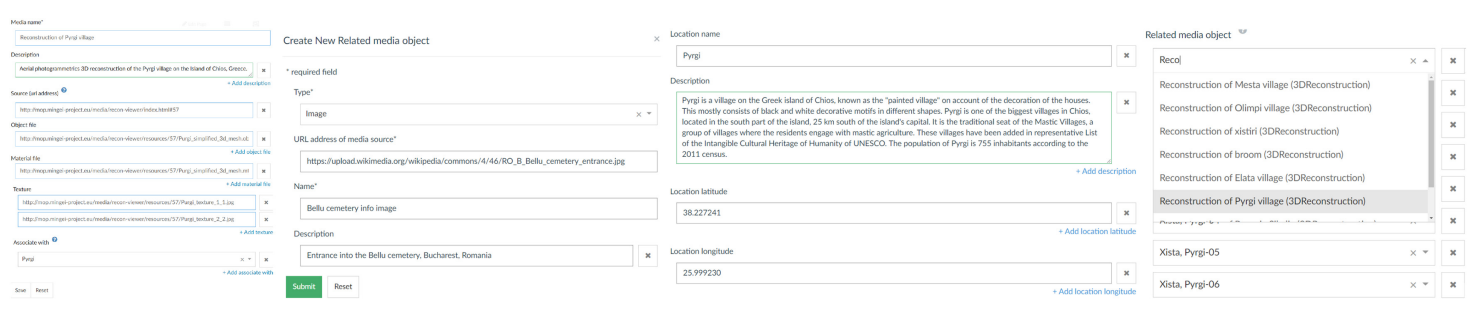

Fig. 6. Forms for instantiating media objects (left half) and knowledge elements (right half).

the Hypertext Transfer Protocol (HTTP). No operation is required for resources already online. When data collection is complete, all media objects are seamlessly treated and their storage location is transparent to the user.

The instantiation of media objects is enabled using forms that simplify asset linking and the entry of metadata. In Figure 6 (left), the user task is illustrated. The images show a media object instantiation form, for a 3D reconstruction. The asset is comprised of multiple files, representing the geometry and visual appearance of the digitised structure. The form enables the entry of the URL, its appellation, and potential relations to existing knowledge entities, such as a place, a person, or an event. During an evaluation, we found that the introduction of media objects is a frequent task when collecting auxiliary data for a knowledge entity. Thus, a User Interface (UI) shortcut was developed for quick asset entry (second from the left image, in Figure 6).

\subsection{Knowledge Representation}

The representation of knowledge elements encompassed is implemented using type-specific data entry forms, as per the knowledge types in Section 3.3. The form for instantiating a "Location" is shown in Figure 6 (second from left). The data entered in the form fields are either literals or links that associate the authored element with others.

For common data types, entry is aided by conventional UI components, i.e., a calendar for dates and a map for locations. For named geographical locations, data entry is integrated with the FactForge service [30], to retrieve the corresponding coordinates from the GeoNames [35] database. Linking of media objects is facilitated by autocomplete, pop-up menus; while the user types, matching media objects dynamically update in a pop-up menu, as in Figure 6 (rightmost). 

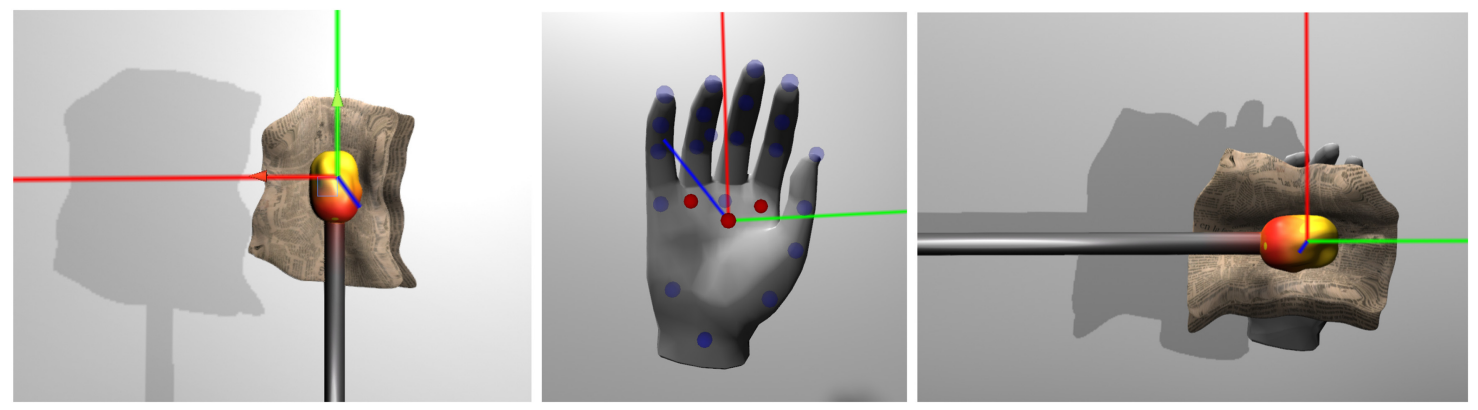

Fig. 7. Visualisation of tool grip annotation.

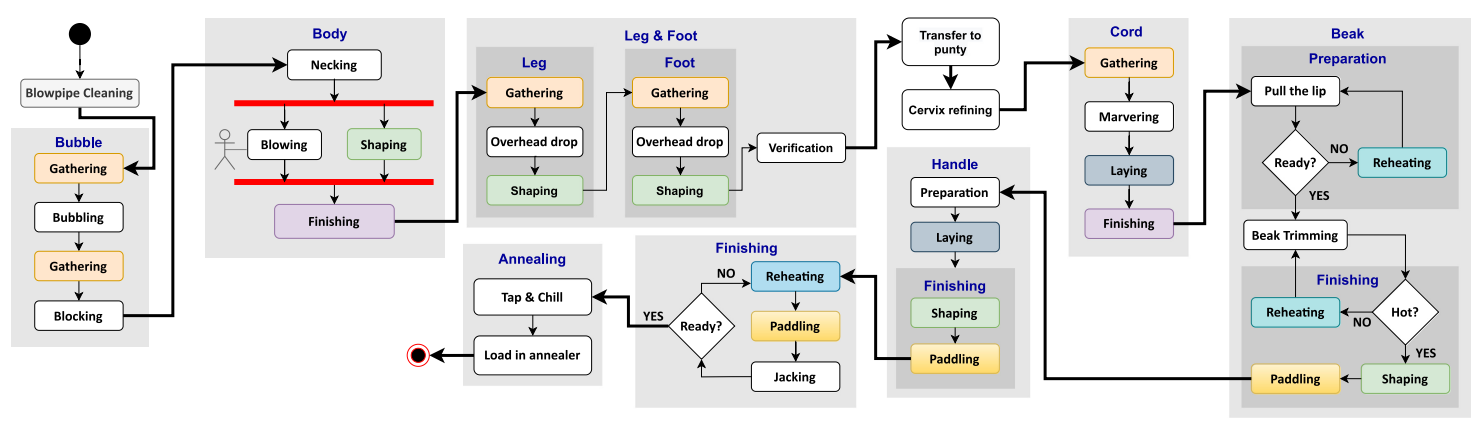

Fig. 8. Activity diagram obtained from ethnography.

From motion recordings, reference postures and gestures are identified. This can be a tedious task as craft demonstration recordings include idle time, mistakes, and repetitions. To associate frames and segments from motion recordings with postures and gestures, respectively, the AnimIO annotation editor [70] was employed. AnimIO facilitates body-member-specific annotation of motion recordings, enabling the selection of body members relevant to a gesture.

To represent tool and machine usage, motion recordings and 3D models are combined. The physical interface of the practitioner with a tool or machine component at a moment in time is represented by a posture and a rigid transformation, a $3 \mathrm{D}$ translation in $\mathbb{R}^{3}$ and a $3 \mathrm{D}$ rotation in $S O(3)$. The posture refers to the practitioner body or one of its members. The transformation is in the coordinate frame of the root node of the body or member. The transformation brings the tool's model to the designated pose relative to the body or member. Its numeric expression is facilitated using the TooltY editor [99]. In Figure 7, this operation is illustrated. Local coordinate frames are shown, as colour lines. On the left, shown is the object and its coordinate frame, centred at the contact point and oriented in a way that prescribes how the object is to be held for a specific gesture by a right-handed person. In the middle image, a right-hand model and coordinate frame are shown. The frame is defined in relation to anatomical landmarks (red points). In the right image, the prescribed tool grip is implemented.

\subsection{Workflow Representation}

An activity diagram is authored, based on the understanding of the crafting process. This task is iterative and reviewed by the practitioners. We used the "diagrams.net" editor [53] to create the diagrams and export them in Extensible Markup Language (XML). The activity diagram for the creation of a glass carafe is shown in Figure 8. The process refers to two persons, having the roles of a master and an assistant. 

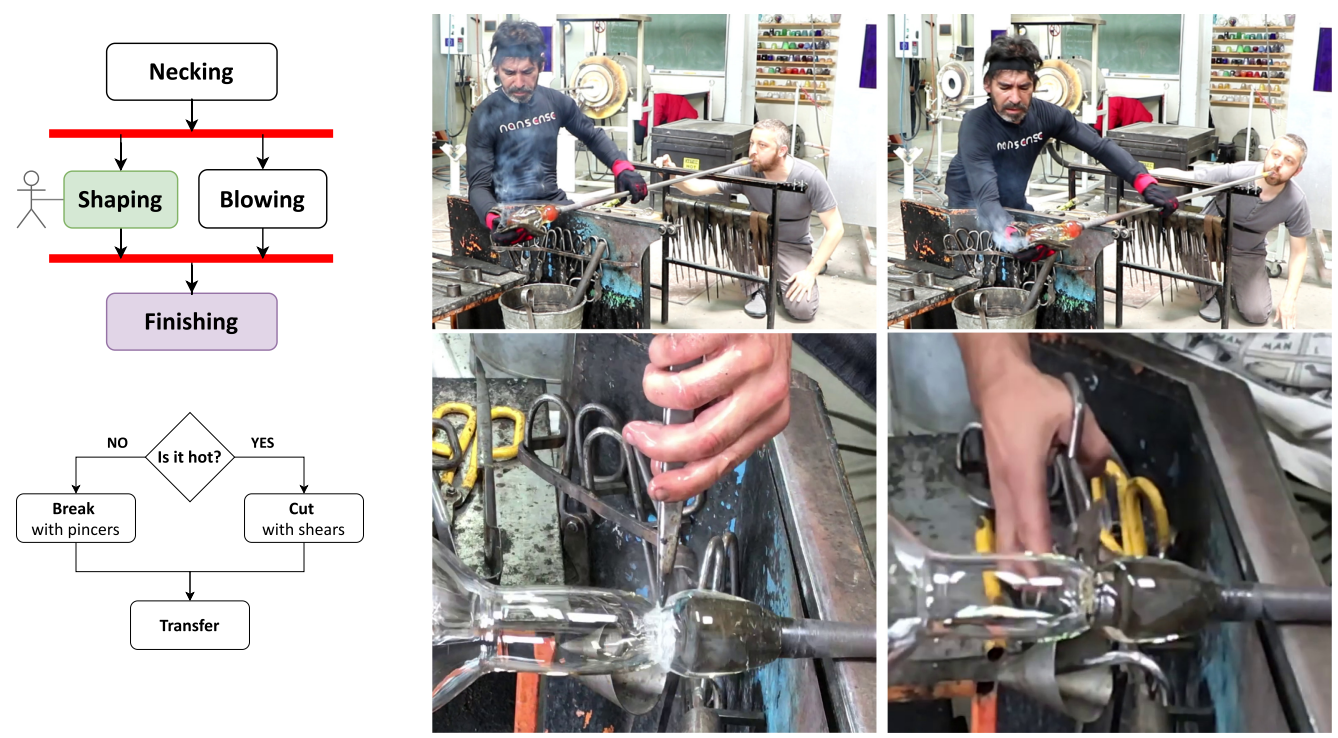

Fig. 9. Activity diagrams and images for steps (top) and a branch step (bottom).

The first step is preparatory and is the cleaning of the blowpipe from past residues. "Bubble" is the step during which a gathering of liquid glass is transformed into a glass bubble. Its (sub) steps are sequentially modelled using instances of class "Transition." If this sequence is analysed in more detail, decision points are observed. For example, cleaning the blowpipe implies the judgement of whether the blowpipe is sufficiently clean and, thus, the use of class "Branch." Recursive refinement of steps is supported by the implementation. The granularity of representation is determined by the curator.

In Figure 9, the treatment of synchronised collaboration and decisions is shown. The top shows a step, where the process is bifurcated into two concurrent flows, each for one person; the stick figure denotes the master's activity flow. The master shapes the blowpipe-attached bubble with a wet newspaper, rolling it back and forth, to counter gravity and enforce symmetry. The assistant blows from the other end, moving in synchrony to the master. Both workflows start and end together. On the bottom, shown is a more detailed analysis of the "Transfer to punty" step, where the practitioner separates the glass body from the blowpipe. The way that the body should be detached from the tip of the blowpipe depends on the viscosity of the bubble, that is, its temperature. If judged to be hot enough, it is cut using a pair of shears (middle), without the danger of breaking. Otherwise, it is carved and broken away from the blowpipe, using a pair of pincers (right).

Activity diagrams were found to be an efficient and intuitive way to collaboratively create visual and unambiguous crafting process representations, used as a map to overview, but also denoting transition types and decision points.

\subsection{Process Schema Representation}

A UI component enables the instantiation of process schemas and their steps. Data fields are used to enter appellations and informal descriptions. Step order is determined by the transitions that link process schema steps or can be explicitly set. Transitions are instantiated via a dynamic UI component that adapts to transition type. At its top, a menu enables the choice of transition type. Once selected, the UI component adapts to offer the transition-specific parameters defined in Table 3. Incoming and outgoing links are instantiated using dynamic menus that contain the names of already defined steps. In Figure 10, these components are shown in the left pair of images. In the leftmost image, shown is the editor's view of a process step schema called and its sub-steps. 


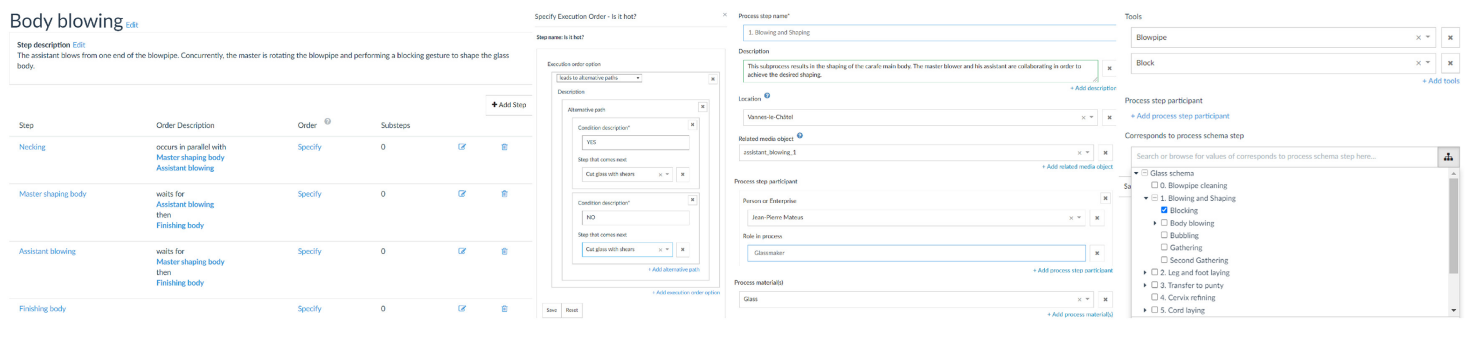

Fig. 10. UI components for authoring representations of process schemas (left half) and processes (right half).

Edit buttons enable the management of the step list and the transition relations between them. The dialogue box on the second from the left image is the UI component for a branch transition.

\subsection{Process Representation}

A set of UI components enable instantiation of processes as activities, via the entry of attribute data, chronological ordering, and the association with recordings that document them. The UI enables linking an arbitrary number of knowledge entities to a process step. It facilitates the task through contextual searches in the form of dynamic, auto-complete, pop-up menus as in Section 4.3, for materials, tools, and participants.

The UI components are shown in the right pair of images, in Figure 10. The UI in the second from the right image enables the linking of processes and process steps with their corresponding process schemas and step schemas. This is implemented by the field "Corresponds To" that is used to associate a process step with a process step schema. The task is facilitated by a dynamic menu that follows the process schema step hierarchy, as shown at the bottom of the rightmost image. As noted in Section 3.6, creating this link is optional. The rationale for this choice is the following. The process schema is an abstraction of all the processes that follow that schema. To achieve this abstraction, multiple process demonstrations may have to be studied. Thus, instantiation of processes without association with a process schema allows for the definition of this schema later on, after sufficient data have been studied.

\section{APPLICATIONS}

Ways to preserve, share, and present the represented content are proposed. The MOP supports third-party applications by responding to Web queries, which retrieve content from this representation. This content is formatted according to the purpose of the retrieval. Variations of export are implemented through templates, which format the output according to prescribed structures and formats. Multiple templates are provided to match specific presentation and preservation needs, in machine-interpretable and human-comprehensible forms.

\subsection{Digital Preservation}

Digital preservation templates export knowledge and assets, in machine-interpretable formats.

5.1.1 Digital Assets. Digital assets are in conventional and open formats. Each asset has a unique Internationalised Resource Identifier (IRI) to be directly integrated by third parties. Assets are accompanied by technical metadata, enabling content type identification and transformation to future formats. A mapping between our ontology and the Europeana Data Model (EDM) [69] enables the utilisation of Europeana assets and the ingestion of new assets in Europeana.

5.1.2 Knowledge Entities. The crafting process representation is digitally preservable in machineinterpretable format. It is encoded in the ontology schema, with semantic links and knowledge statements stored in RDF. The RDF guarantees syntactical interoperability and can be shared across implementations. Semantic 


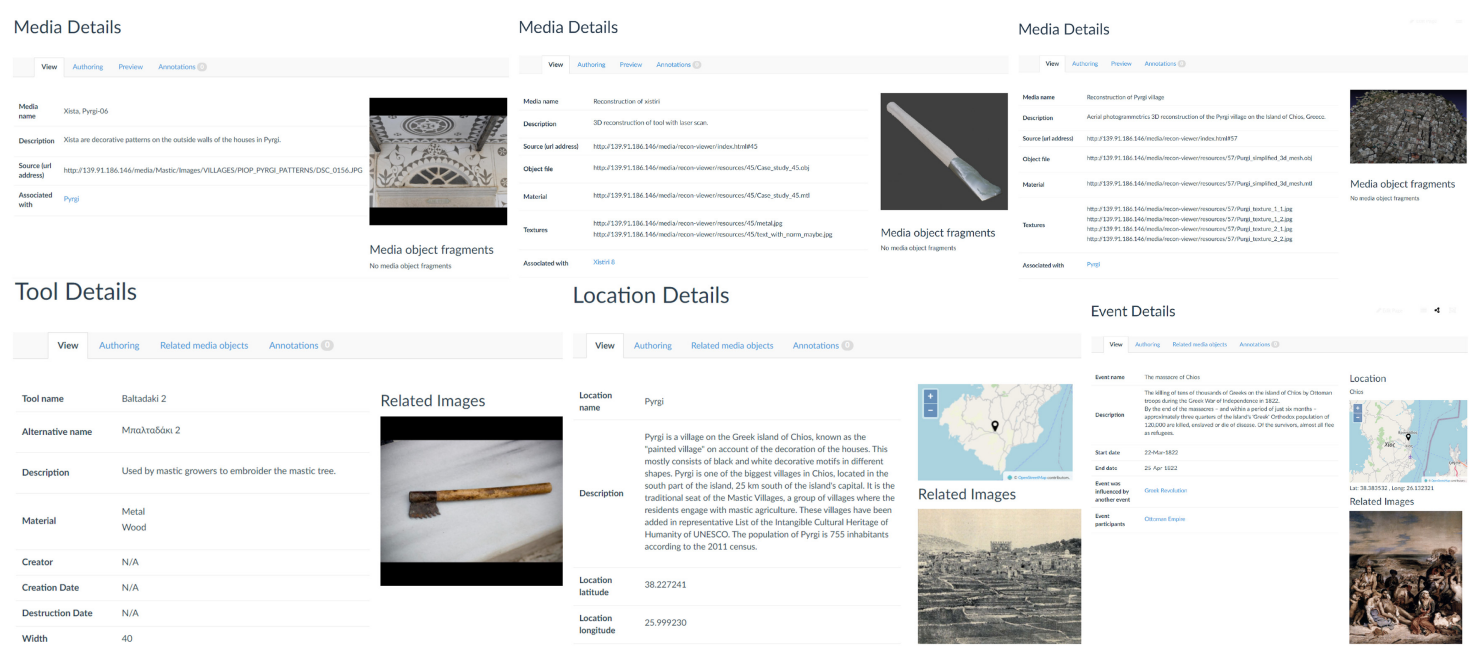

Fig. 11. Documentation pages for media objects (top) and knowledge elements (bottom).

interoperability is guaranteed by the use of an ontology that is based on the CRM. The MOP maintains a representation that might be continuously updated. To refer to specific versions of this representation, the RDF export can be stored in any conventional versioning system.

\subsection{Documentation}

A human-comprehensible way to present the represented knowledge network is hypertext. The implementation employs a Web interface that dynamically generates Hypertext Markup Language (HTML) pages from the knowledge queries and a Web server that transmits them to the Web client (browser). An individual documentation page is provided for each entity. Semantic links are implemented as hyperlinks that lead to the pages of cited entities. This way, browsing and exploration of knowledge through semantic associations are enabled. Contents can be organised and presented spatiotemporally or thematically. A keyword-based search is provided.

Documentation pages for media objects contain links to digital assets, textual presentation of metadata, and previews of the associated digital assets. One or more URLs are provided on each page. For media objects, these links point to the source data files. For knowledge entities, the link points to the RDF encoding of that entity. For locations and events, specific UI modules are provided. For locations, embedded, dynamic maps are provided through OpenStreetMap [32]. Time-line and calendar views are available for events.

5.2.1 Media Objects. The documentation pages for media objects contain links to digital assets, metadata, and previews of the digital assets. Some assets are comprised of multiple files, e.g. 3D reconstructions contain geometry and texture files, videos are accompanied by multiple subtitles, and so on. For video, MoCap, and 3D models, embedded viewers are provided. In Figure 11 (top), examples of pages for are shown for a photograph [78], a 3D model of a tool [77], and a 3D reconstruction of a village [76].

5.2.2 Knowledge Entities. Documentation pages for knowledge elements are type-specific, as per the types in Section 3.3. Pages for instances of class "Person" provide biographical information and a portrait image. "Location" instances contain maps and, if any, the previews of associated digital assets. The pages for a tool [73], a location [75], and an event [74], are shown in Figure 11 (bottom). In reciprocity, the pages for media objects include hyperlinks to the pages of the knowledge elements that refer to them. For example, the page for the 3D reconstruction of the Pyrgi village contains a hyperlink to the documentation page for the Pyrgi location. 


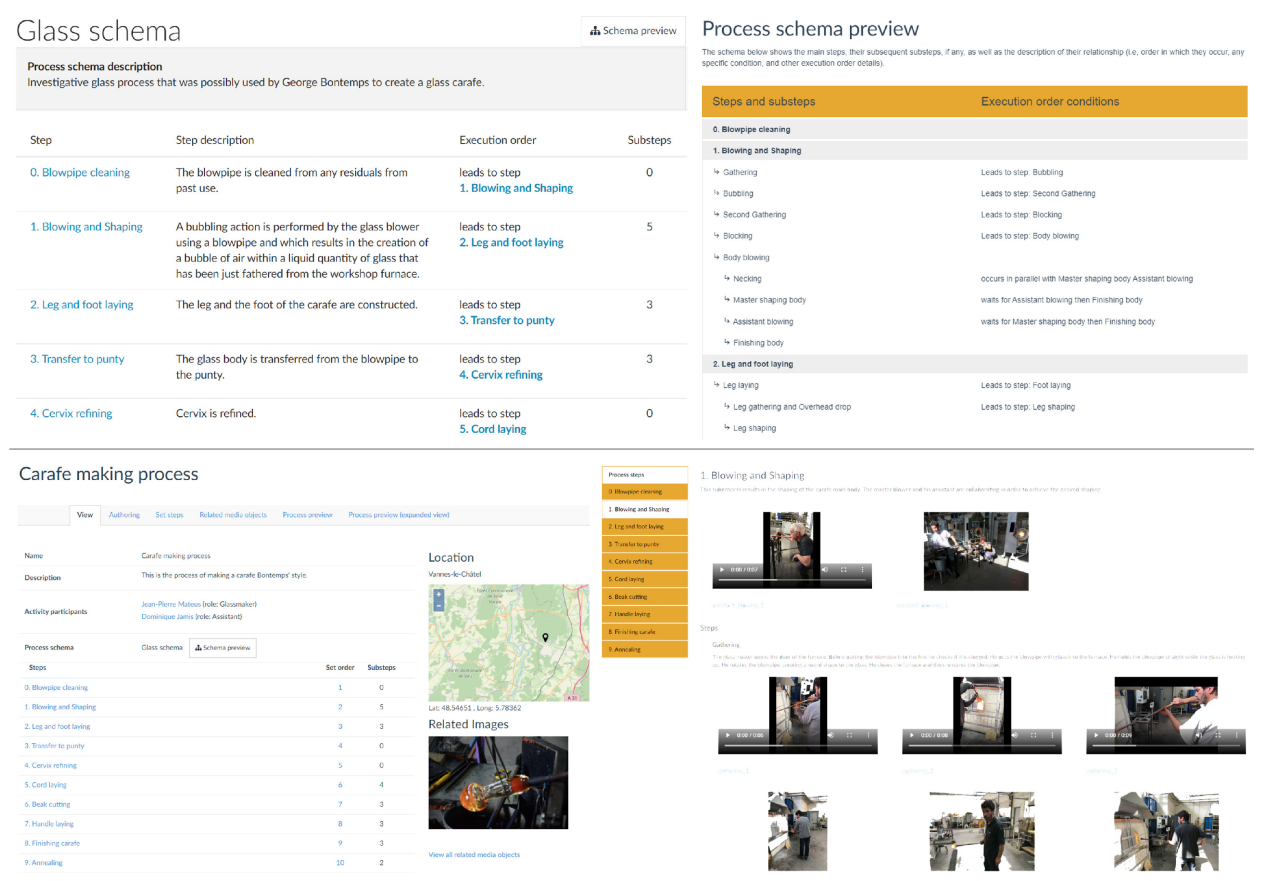

Fig. 12. Documentation pages for process schemas (top) and processes (bottom).

5.2.3 Vocabularies. An output of this organisation of knowledge is illustrated vocabularies of tools, which bring together verbal descriptions and visual recordings. In the same way, the steps where a specific tool is used can be retrieved, along with video recordings of such actions; and similarly, for the tools and materials required for a certain process. An example is provided for the case of Glass tools. Queries that exhaustively retrieve members of semantic classes are used to support browsing and cataloguing.

5.2.4 Process Schemas. Documentation pages for process schemas show step schemas and their relations using hypertext. A serial presentation of the step hierarchy is provided, as tabulated text. In Figure 12 (top), the documentation page for a process schema [80] and its tabulated preview are shown.

5.2.5 Processes. Processes are presented as a series of events, while also denoting cases of concurrent activities. Events are knowledge entities and are presented on an individual documentation page each. The process step documentation page is a specialisation of the generic documentation page for instances of class "E5 Event." It contains links to the recordings of the knowledge elements for the tools and materials involved, the participating practitioners, the date, and the location of the recording. If the process follows a process schema, a link to that schema and its preview are also provided. The hierarchy of process steps is presented using insets, each one presenting textual information and previews of the available digital assets. To present step organisation, insets can be dynamically unfolded to any depth of the process hierarchy. Each inset dynamically unfolds and each step is associated with image previews and embedded videos. An index of the first level of process steps of the hierarchy is provided on the left. The result is a multimedia-enriched semantic presentation of the process. In the right images of the bottom row, shown is one out of a handful of implemented configurations. Variations include images and textural descriptions. The main documentation page for a process [79] is shown in Figure 12 (bottom), followed by a presentation page that is configured to show a video synopsis of the process on the top of the page and, below, the video synopses of steps. 

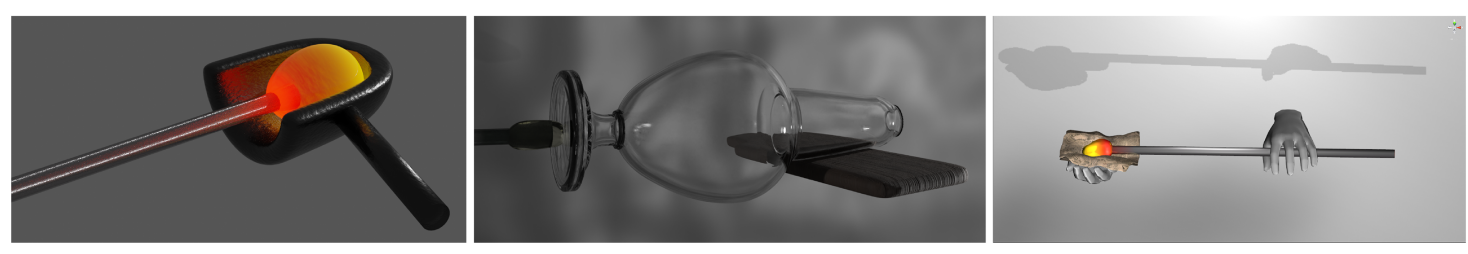

Fig. 13. Visualisations of tool and hand postures.

\subsection{Visualisation and Storytelling}

An added value of the proposed approach is found in the presentation of the contents of the knowledge base. As process steps are associated with both signal and semantic descriptions, they can be presented together, the signal components used to show recordings of events and the semantic components to interpret them. Using visualisation and storytelling tools knowledge encoded in the representation can be explained from multiple perspectives.

5.3.1 Verbal Descriptions. Written or spoken descriptions are employed to assist comprehension of each entity. For each step process step and transition in between, the same textual descriptions as in Section 5.2 are available, as well as those involved in each step. When authoring the process presentations, the individual step descriptions are available for the curator to modify them, if needed, in the context of each specific presentation.

5.3.2 Postures. The selection of keyframes in posture documentation comprises knowledge abstractions that can be used in exemplars [1]. Posture visualisation is available through avatars, using AnimIO [70]. The digitised 3D tool model is aligned with the avatar hand reference frame, using the stored grip posture. This way, tool grip, relative configuration, and motion are rendered in 3D, enabling close inspection and viewpoint of choice. In Figure 13, the relative placement of glasswork tools (left, middle) and the bimanual posture (right) from the task shown in Figure 9 (top) are shown.

5.3.3 Animated Gesture Presentation. Similarly to Section 5.3.2, 3D motion and posture recordings are retrieved from the knowledge base. Using AnimIO [70], they are mapped to avatars and digitised objects, respectively, to create animated 3D gesture presentations. The 3D animations are automatically produced, mapping the recorded movements to avatars and using the recorded postures for handled tools and objects. The 3D animations can be viewed immersively or rendered as a conventional video.

5.3.4 Pictorial Gesture Presentation. The juxtaposition of keyframes in chronological order guides inference of what occurred in between them, while motion annotation facilitates comprehension of the recorded movement even in single frames [58]. The MoTiVo annotation editor [91] provides motion line [27] annotation. Textual annotations can be added. In this work, the interactive segmentation method in [93] was integrated to guide perceptual figure/ground assignment [71] to regions of interest, through the simplification of background image structures. In Figure 14(a), top row, three background-simplified key video frames from a video are shown in temporal order, illustrating the gesture required to make a carafe handle. In Figure 14(a), bottom row, are shown motion annotations with ethnographic findings as textual annotation, compiled in a graphic description of the process [90].

5.3.5 Processes. The representation of crafting processes is presented in chronological order, interpreting the causal relations between steps as conditional transitions. Depending on the type of presentation medium, i.e., verbal, pictorial, and animated, the corresponding metadata are retrieved and presented as in Sections 5.3.4 and 5.3.3, respectively. The storyboard for the glasswork examples in this work was compiled in a booklet [90] and integrated with an MR system [56], as shown in Figure 14. Triggered by touch, pictorial presentations are associated with original videos and explanatory narration [83]. In Figure 15 (top), gesture animations synthesise a motion-driven, visual narrative, interactively visible from the viewpoint of choice or exported in video. In Figure 15 (bottom), the same method is applied to the recorded motion of a mastic cultivator to demonstrate the 

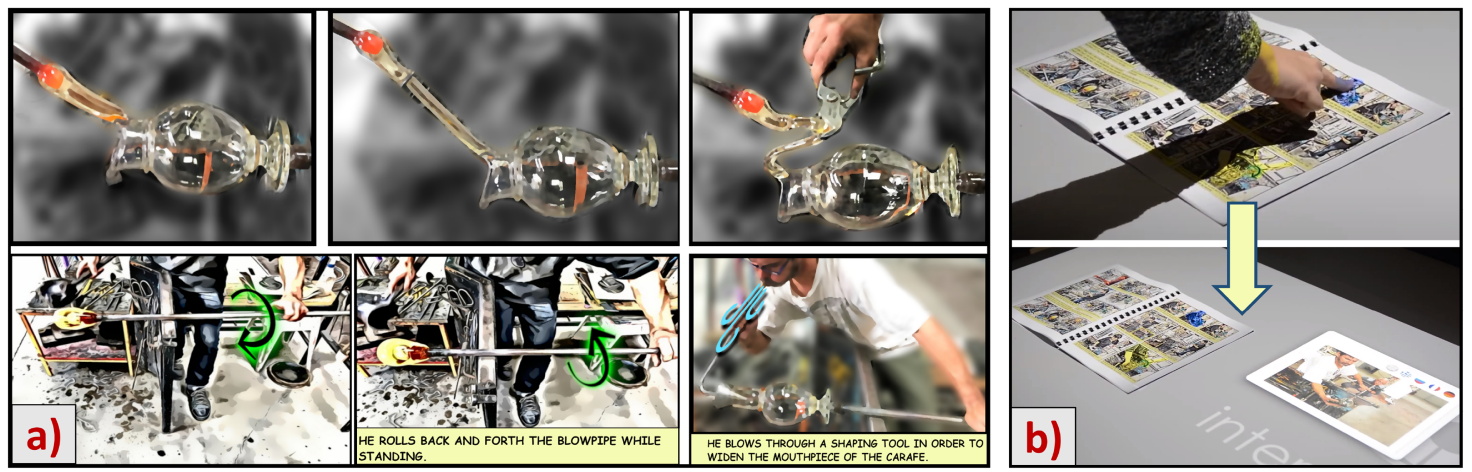

Fig. 14. (a) Illustration of glasswork gestures, (b) MR glasswork presentation.
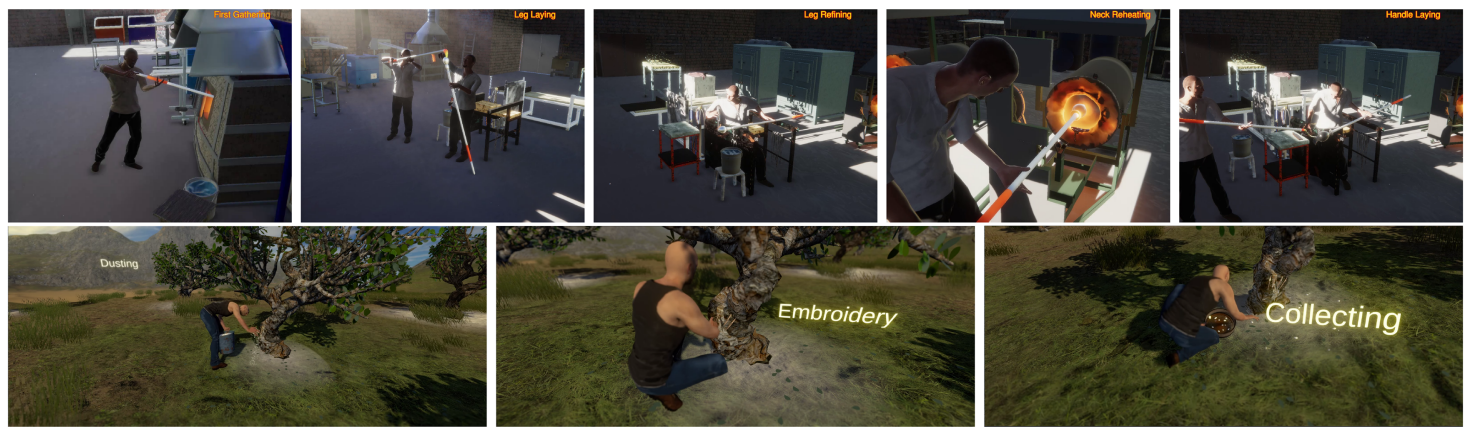

Fig. 15. 3D glasswork (top) and mastic cultivation (bottom) animations.

cultivation process; the video can be found in [84]. These presentations are semi-automatically produced, in that the animations produced in Section 5.3.3 have to be placed and oriented in the simulated environment. As in Section 4.3, the TooltY editor [99] is used to define this rigid transformation.

5.3.6 Workspaces. Registering objects and processes upon the spatial reference of the workshop enables explanatory presentations. In Figure 16 (left), shown are first-person views of a glass workshop and its equipment. To understand the relations between the body, the tools, the material, and the workspace, we used the framework of "operational sequences" [52] and mapped practitioner movement is mapped to the 3D model of the workshop. Analysed MoCap data are visualised in Figure 16 (right), mapping the walking path of the practitioner on a 3D model of a glass workshop (red, dashed line). The data pertain to the transfer of a gather of liquid glass from the furnace and the return to the workbench (blue oval). The walkthrough corridor is broad, to accommodate multiple practitioners. The distance of the bench to a couple of glory holes (green dots) is small and allows easy access to, the frequent task of, glass reheating. The representation of the environment illumination can provide experiential information. In Figure 15, we simulated time and weather, as mastic collection takes place in early September. Sunshine and high temperature are the norms during this season and, thus, mastic collection initiates before sunrise and ends before noon.

\section{CONCLUSION AND FUTURE WORK}

A method that leads to the representation and digital preservation of traditional crafting processes is proposed. This includes the identification and organisation of data and knowledge that document and interpret these 


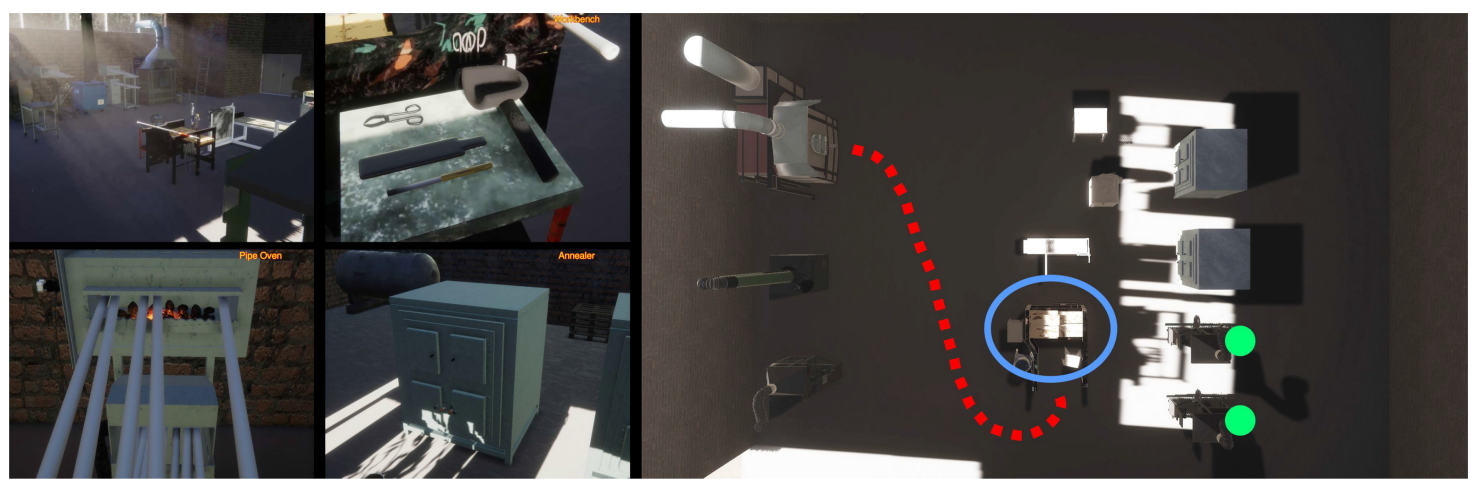

Fig. 16. First-person (left) and annotated, top-view (right) of a 3D model of a glass workshop.

processes. The representation accommodates both signal and semantic dimensions of this content, relating recordings with their interpretations, in a new semantic model. The proposed approach stands on the legacy of digital documentation and preservation of $\mathrm{CH}$. The outcome representation is compatible with contemporary digital preservation standards. The implementation is provided as an online, multiple user platform, accompanied by auxiliary tools. Applications are demonstrated in digital preservation and presentation of $\mathrm{CH}$ due to crafts.

No practice can be mastered without (a lot of) practice. The provided approach provides documentation, but which cannot substitute the experience needed to master a craft. The purpose of providing clear documentation of materials and methods and insightful instructions is to support the repeatability of the recorded practice. "Felt information," or qualia [18], are used in craft practice. Examples are haptic sensations, weight and balance, viscosity, and other percepts [43] that the practitioner exploits to take decisions on the fly. Tacit [81] or embodied [101] knowledge refers to learned interpretations of stimuli and experienced performance of appropriate actions that are not simple to verbalise or visualise. Another consideration is that perception is active, in that attention and sensitivity to stimuli adapt to the purpose of observation [5]. 


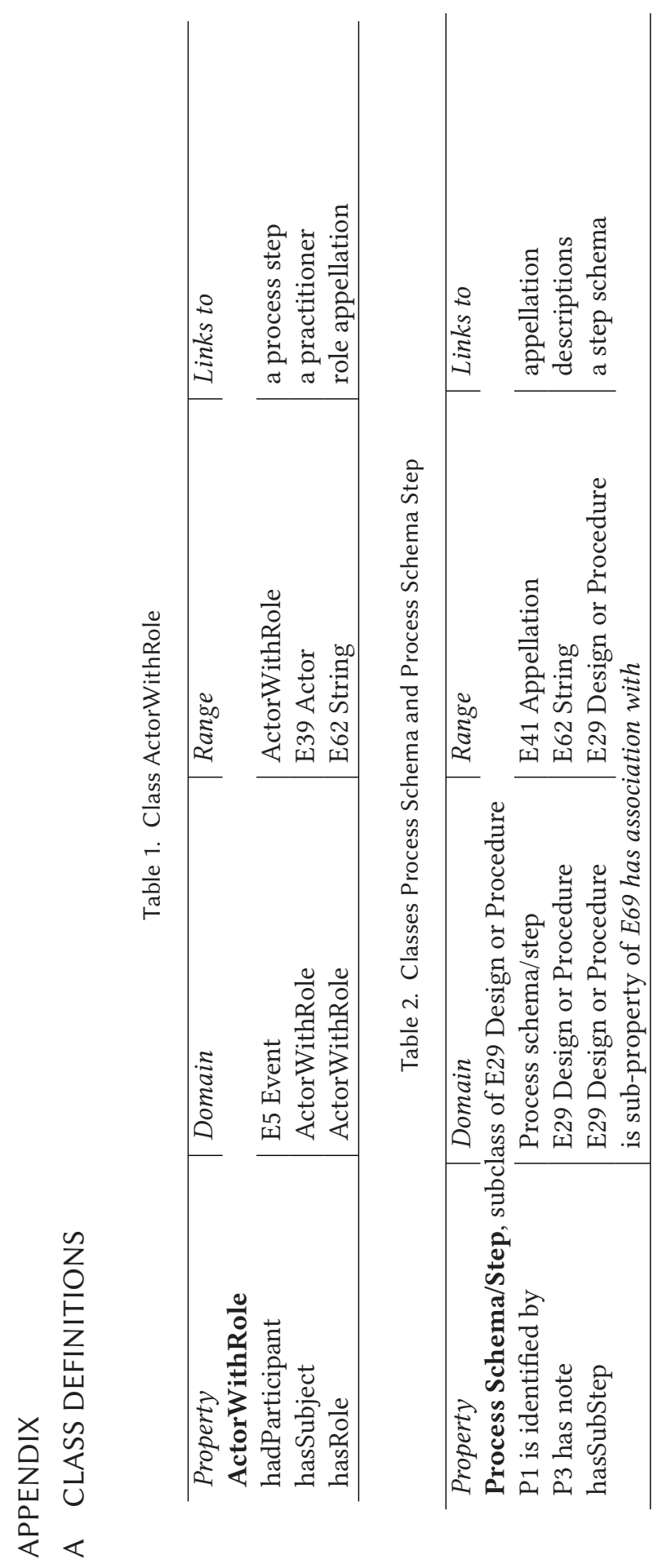




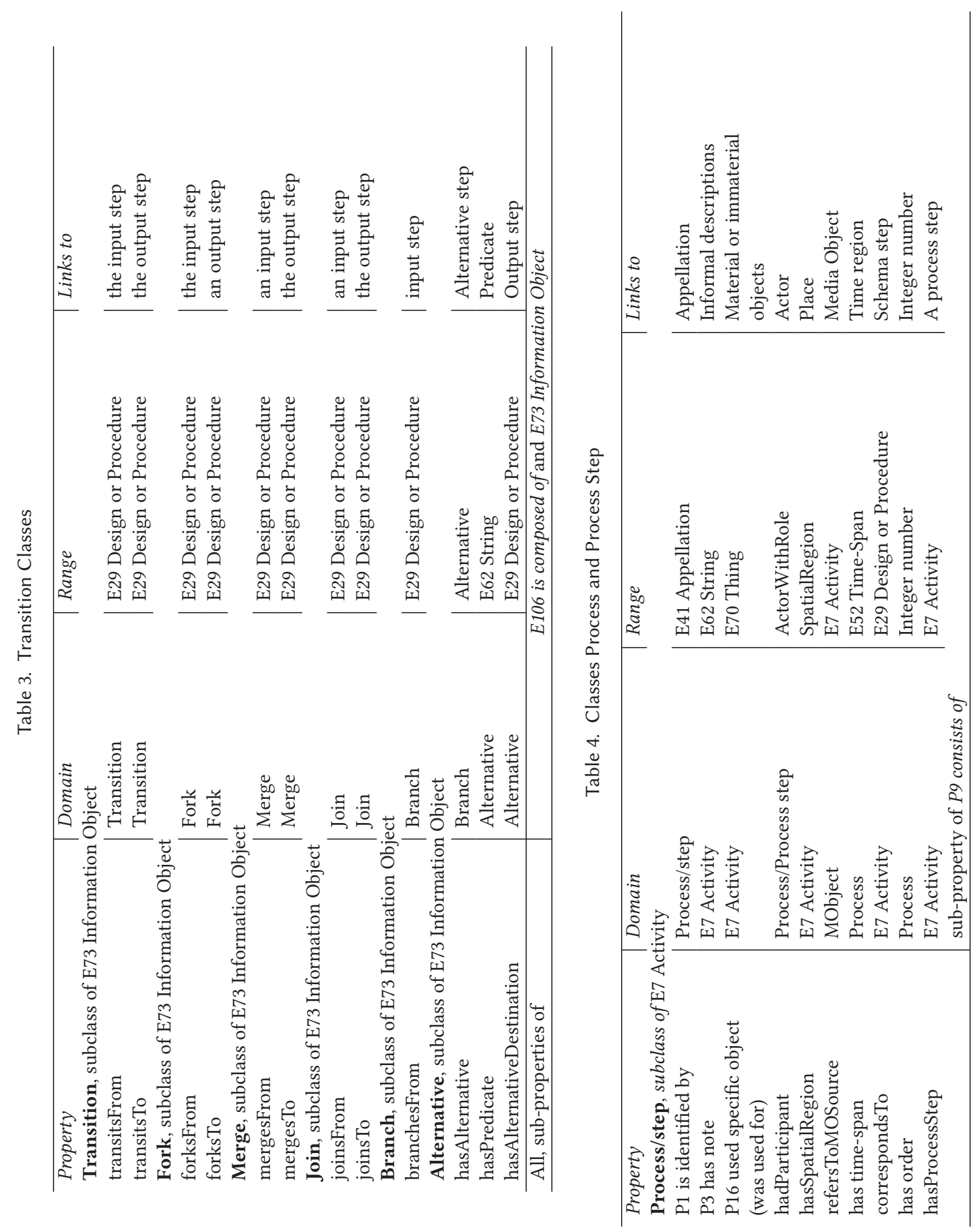

ACM Journal on Computing and Cultural Heritage, Vol. 15, No. 3, Article 53. Publication date: September 2022. 


\section{ACKNOWLEDGMENTS}

The authors are grateful to the anonymous reviewers whose constructive criticism enabled the significant improvement of this manuscript.

\section{REFERENCES}

[1] G. Almevik and K. Melin. 2016. Conservation theory for enhanced craft practice. In International Conference on Structural Analysis of Historical Constructions. CRC Press, Leuven, Netherlands, 1011-1018.

[2] R. Arp, B. Smith, and Spear A. 2015. Building Ontologies with Basic Formal Ontology. MIT Press, Cambridge, MA.

[3] P. Atkinson. 1990. The Ethnographic Imagination: Textual Constructions of Reality. Routledge, London, UK. https://doi.org/10.4324/ 9781315852065

[4] P. Atkinson. 2013. Blowing hot: The ethnography of craft and the craft of ethnography. Qualitative Inquiry 19, 5 (2013), $397-404$. https://doi.org/10.1177/1077800413479567

[5] R. Bajcsy. 1988. Active perception. Proceedings of the IEEE 76, 8 (1988), 966-1005. https://doi.org/10.1109/5.5968

[6] G. Barra. 2012. Chinese Craft Project. Master's thesis. Politecnico Di Milano, Milano.

[7] E. Benetos, S. Dixon, Z. Duan, and S. Ewert. 2019. Automatic music transcription: An overview. IEEE Signal Processing Magazine 36, 1 (2019), 20-30. https://doi.org/10.1109/MSP.2018.2869928

[8] R. Bloomberg, M. Dekkers, S. Gradmann, M. Lindquist, C. Meghini, and J. Verleyen. 2010. Functional specification for the Europeana Danube release. http://pro.europeana.eu/documents/844813/851994/D3_2_Final.pdf. Accessed on 2 May 2021.

[9] W. Bosma, P. Vossen, A. Soroa, G. Rigau, M. Tesconi, A. Marchetti, M. Monachini, and C. Aliprandi. 2009. KAF: A generic semantic annotation format. In GL2009 Workshop on Semantic Annotation. International Committee on Computational Linguistics, Centre Universitaire, Luxembourg, 1-8.

[10] C. Brown, J. Hicks, C. Rinaudo, and R. Burch. 2021. The use of augmented reality and virtual reality in ergonomic applications for education, aviation, and maintenance. Ergonomics in Design 1 (March 2021), 10648046211003469. https://doi.org/10.1177/10648046211003469

[11] E. Ceseracciu, Z. Sawacha, and C. Cobelli. 2014. Comparison of markerless and marker-based motion capture technologies through simultaneous data collection during gait: Proof of concept. PLoS One 9, 3 (March 2014), 1-7. https://doi.org/10.1371/journal.pone. 0087640

[12] D. Chaffin. 2007. Human motion simulation for vehicle and workplace design. Human Factors and Ergonomics in Manufacturing \& Service Industries 17, 5 (2007), 475-484. https://doi.org/10.1002/hfm.20087

[13] F. Cominelli. 2011. Governing Cultural Commons: The case of traditional craftsmanship in France. In Biennial Conference. International Association for the Study of the Commons, Hyderabad, India, 1-27.

[14] A. Corns. 2013. 3D-ICONS: Guidelines and Case Studies. 3D-ICONS was a project funded under the European Commission's ICT Policy Support Programme, Project No. 297194. https://doi.org/10.5281/zenodo.1311797

[15] R. Cyganiak, D. Wood, and M. Lanthaler. 2014. RDF 1.1 Concepts and Abstract Syntax. W3C Recommendation. WWW Consortium. http://www.w3.org/TR/rdf11-concepts/. Accessed on 2 May 2021.

[16] G. de Francesco. 2004. MINERVA: The ministerial network for valorising activities in digitisation towards an agreed European platform for digitisation of cultural and scientific heritage. International fournal Information Theories and Applications 11 (2004), 240-247.

[17] S. de los Ríos, M. Cabrera-Umpiérrez, M. Arredondo, P. Abril, V. Jiménez, and C. Giachritsis. 2014. Using cloud technologies for engaging people with cultural heritage. In International Conference on Universal Access in Human-Computer Interaction. Springer International Publishing, Wiesbaden, Germany, 673-680.

[18] D. Dennett. 1988. Quining qualia. In Consciousness in Contemporary Science, A. Marcel and E. Bisiach (Eds.). Oxford University Press, Oxford, 42-77.

[19] Haus der Seidenkultur. 2018. Hanging on a thread. Video, G. Oehms, M. Weisters, R. Viehweg-Weber, W. Volker. https://youtu.be/ dahgDvtSCdI. Accessed on 2 May 2021

[20] K. Dimitropoulos, S. Manitsaris, F. Tsalakanidou, S. Nikolopoulos, B. Denby, Al K., L. Crevier-Buchman, C. Pillot-Loiseau, M. AddaDecker, S. Dupont, J. Tilmanne, M. Ott, M. Alivizatou, E. Yilmaz, L. Hadjileontiadis, V. Charisis, O. Deroo, A. Manitsaris, I. Kompatsiaris, and Grammalidis N. 2014. Capturing the Intangible: An introduction to the i-Treasures project. In International Conference on Computer Vision Theory and Applications. Institute of Electrical and Electronics Engineers, Lisbon, Portugal, 773-781.

[21] M. Doerr. 2003. The CIDOC conceptual reference module: An ontological approach to semantic interoperability of metadata. AI Magazine 24, 3 (Sept. 2003), 75-92.

[22] M. Doerr, C. Bekiari, and P. LeBoeuf. 2008. FRBRoo, a conceptual model for performing arts. In 2008 Annual Conference of CIDOC. CIDOC - ICOM International Committee for Documentation, Athens, Greece, 06-18.

[23] M. Doerr, S. Gradmann, S. Hennicke, A. Isaac, C. Meghini, and H. van de Sompel. 2010. The Europeana Data Model (EDM). In World Library and Information Congress: General Conference and Assembly. International Federation of Library Associations, Hague, Netherlands, $10-15$.

[24] L. Donkin. 2001. Crafts and Conservation. Report. ICCROM. 
[25] S. Doolani, L. Owens, C. Wessels, and F. Makedon. 2020. vIS: An immersive virtual storytelling system for vocational training. Applied Sciences 10, 22 (2020), 1-15. https://doi.org/10.3390/app10228143

[26] A. Doulamis, A. Voulodimos, N. Doulamis, S. Soile, and A. Lampropoulos. 2017. Transforming intangible folkloric performing arts into tangible choreographic digital objects: The terpsichore approach. In International foint Conference on Computer Vision, Imaging and Computer Graphics Theory and Applications, Vol. 5. INSTICC, Porto, Portugal, 451-460. https://doi.org/10.5220/0006347304510460

[27] M. Edwards and M. Crane. 2007. Motion streaks improve motion detection. Vision Research 47, 6 (2007), 828-833.

[28] T. Evdemon, A. Katzourakis, and X. Zabulis. 2021. Mitata at Psiloritis UNESCO Global Geopark. Dataset. https://doi.org/10.5281/zenodo. 4447174

[29] T. Evdemon, N. Patsiouras, and X. Zabulis. 2021. Angel, Yannoulis Chalepas, https://doi.org/10.5281/zenodo.4474932 1875. Accessed on 2 May 2021.

[30] FactForge. 2021. FactForge Open Data and News about People, Organizations and Locations. http://factforge.net/. Accessed on 2 May 2021.

[31] M. Field, D. Stirling, F. Naghdy, and Z. Pan. 2009. Motion capture in robotics review. In IEEE International Conference on Control and Automation. Institute of Electrical and Electronics Engineers, Christchurch, New Zealand, 1697-1702. https://doi.org/10.1109/ICCA. 2009.5410185

[32] Open Street Map Foundation. 2021. Open Street Map. https://www.openstreetmap.org/. Accessed on 2 May 2021.

[33] O. Samuelsson, G. Almevik, and P. Jarefjäll. 2013. Tacit record: Augmented documentation methods to access traditional blacksmith skills. In International Conference on Design and Digital Heritage. University of Gothenburg, Stockholm, 143-160.

[34] C. Geertz. 1973. The Interpretation of Cultures, Vol. 5019. Basic Books, New York.

[35] GeoNames. 2021. GeoNames Geographical Database. https://www.geonames.org/. Accessed on 2 May 2021.

[36] J. Gibson. 1966. The Senses Considered as Perceptual Systems. Houghton Mifflin, Boston.

[37] Object Management Group. 2007. OMG Unified Modelling Language (OMG UML), Superstructure Version 2.1.1. Specification formal/200702-05. W3C. https://www.omg.org/spec/UML/. Accessed on 2 May 2021.

[38] N. Guarino. 1998. Formal Ontology in Information Systems: Proceedings of the 1st International Conference. IOS Press, Netherlands.

[39] M. Hall, E. Agirre, N. Aletras, R. Bergheim, K. Chandrinos, P. Clough, S. Fernando, K. Fernie, P. Goodale, J. Griffiths, et al. 2012. PATHSExploring digital cultural heritage spaces. In International Conference on Theory and Practice of Digital Libraries. Springer, 500-503.

[40] C. Hampson, M. Agosti, N. Orio, E. Bailey, S. Lawless, O. Conlan, and V. Wade. 2012. The CULTURA project: Supporting next generation interaction with digital cultural heritage collections. In Euro-Mediterranean Conference. Springer, Berlin, 668-675.

[41] S. Harris and A. Seaborne. 2008. SPARQL 1.1 Query Language. W3C Recommendation 21 March 2013. W3C. http://www.w3.org/TR/ sparql11-query/. Accessed on 2 May 2021.

[42] J. Hobbs and F. Pan. 2020. Time ontology in OWL. W3C Candidate Recommendation. World Wide Web Consortium. https://www.w3. org/TR/owl-time. Accessed on 2 May 2021.

[43] E. Hutten. 1947. Perception and knowledge. The fournal of Philosophy 44, 4 (1947), 85-97. http://www.jstor.org/stable/2019459.

[44] H. Jennings. 2012. Towards a Definition of Heritage Craft. Prepared for Creative and Cultural Skills.

[45] E. Jocelyne. 1990. Methodological Guide to the Collection of Data on Crafts. Technical Report. UNESCO, Paris, France.

[46] P. Kahle, S. Colutto, G. Hackl, and G. Mühlberger. 2017. Transkribus - A service platform for transcription, recognition and retrieval of historical documents. In IAPR International Conference on Document Analysis and Recognition, Vol. 4. Institute of Electrical and Electronics Engineers, 19-24. https://doi.org/10.1109/ICDAR.2017.307

[47] S. Kim, D. Im, J. Lee, and H. Choi. 2019. Utility of digital technologies for the sustainability of Intangible Cultural Heritage in Korea. Sustainability 11, 21 (2019), 1-29. https://doi.org/10.3390/su11216117

[48] N. Kokolantonakis. 2020. Requirements and Protocol of the 3D Documentation of the Heritage Crafts Tools. Dataset. https://doi.org/ 10.5281/zenodo.3813287

[49] K. Konstantinou and A. Anagnostopoulos. 2019. Interweaving contemporary art and traditional crafts in ethnographic research. Art/Research International: A Transdisciplinary fournal 4, 1 (Feb. 2019), 58-82. https://doi.org/10.18432/ari29420

[50] S. Le Bellu and B. Le Blanc. 2012. How to characterize professional gestures to operate tacit know-how transfer. Electronic fournal of Knowledge Management 10, 2 (2012), 142-153. https://hal.archives-ouvertes.fr/hal-00984750.

[51] F. Lenzerini. 2011. Intangible cultural heritage: The living culture of peoples. European fournal of International Law 22 (2011), 101-120.

[52] A. Leroi-Gourhan. 2018. Gesture and Speech. MIT Press, Cambridge, MA.

[53] JGraph Ltd. 2021. diagrams.net diagram editor. https://www.diagrams.net/. Accessed on 2 May 2021.

[54] Boris M., P. Patel-Schneider, and B. Parsia. 2012. OWL 2 Web Ontology Language Structural Specification and Functional-Style Syntax (2nd ed.). W3C Recommendation. W3C. http://www.w3.org/TR/2012/REC-owl2-syntax-20121211/. Accessed on 2 May 2021.

[55] H. Marchand. 2017. Craftwork as Problem Solving. Routledge, London, UK.

[56] G. Margetis, X. Zabulis, S. Ntoa, P. Koutlemanis, E. Papadaki, M. Antona, and C. Stephanidis. 2015. Enhancing education through natural interaction with physical paper. Universal Access in the Information Society 14, 3 (Aug. 2015), 427-447. https://doi.org/10.1007/s10209014-0365-0

[57] M. Mauss. 1967. Manuel d'ethnographie. Payot \& Rivages, Paris, France.

ACM Journal on Computing and Cultural Heritage, Vol. 15, No. 3, Article 53. Publication date: September 2022. 
[58] S. McCloud. 1993. Understanding Comics: The Invisible Art. Tundra Publishing Ltd, Northampton, Massachusetts.

[59] C. Meghini, V. Bartalesi, and D. Metilli. 2021. Representing narratives in digital libraries: The narrative ontology Article. Semantic Web Pre-press, Pre-press (2021), 1-24.

[60] C. Meghini, V. Bartalesi, D. Metilli, E. Karouzaki, V. Doulgeraki, and N. Partarakis. 2020. Mingei Craft Ontology and Online Authoring Platform. Mingei project deliverable D3.4, April 2020. Mingei H2020 Innovation Action. https://www.mingei-project.eu/dissemination/ public-deliverables/. Accessed on 2 May 2021.

[61] C. Meghini, V. Bartalesi, D. Metilli, N. Partarakis, and X. Zabulis. 2020. Mingei Ontology. https://doi.org/10.5281/zenodo.3742829

[62] C. Meghini, D. Giaretta, L. Fusco, S. Ross, M. Guercio, M. Hernandez, U. di Giammatteo, Z. Bjelogrlic, D. Naor, S. Boi, D. Teruggi, K. Ng, L. Briguglio, V. Christophides, B. Bachimont, H. Vinetand, and P. Sedlak. 2007. CASPAR project and the validation of digital preservation techniques. In PV-2007: Ensuring the Long-Term Preservation and Value Adding to Scientific and Technical Data. European Space Agency, Weßling, Germany, 1-14.

[63] French Republic Ministry of Culture. 2008. The national inventory of intangible cultural heritage. https://www.culture.gouv.fr/Sitesthematiques/Patrimoine-culturel-immateriel/La-politique-du-Patrimoine-culturel-immateriel/L-inventaire-national-du-Patrimoineculturel-immateriel. Accessed on 2 May 2021.

[64] Goverment of India Ministry of Textiles and Ministry of Textiles. 2021. Development Commissioner (Handicrafts). Retrieved from http://www.handicrafts.nic.in/. Accessed on 2 May 2021.

[65] S. Mokhov, A. Kaur, M. Talwar, K. Gudavalli, M. Song, and S. Mudur. 2018. Real-Time motion capture for performing arts and stage. In ACM SIGGRAPH 2018 Educator's Forum (Vancouver, British Columbia, Canada) (SIGGRAPH'18). Association for Computing Machinery, New York, NY, Article 4, 2 pages. https://doi.org/10.1145/3215641.3215642

[66] D. Mueller and J. Ferreira. 2003. MARVEL: A mixed reality learning environment for vocational training in mechatronics. In Technology Enhanced Learning International Conference. Institute of Electrical and Electronics Engineers, Milan, Italy, 1-8.

[67] J. Osterlund and B. Lawrence. 2012. Virtual reality: Avatars in human spaceflight training. Acta Astronautica 71 (2012), 139-150. https: //doi.org/10.1016/j.actaastro.2011.08.011

[68] P. Panteleris, I. Oikonomidis, and A. Argyros. 2018. Using a single RGB frame for real time 3D hand pose estimation in the wild. In IEEE Winter Conference on Applications of Computer Vision. IEEE, 436-445. https://doi.org/10.1109/WACV.2018.00054

[69] N. Partarakis, P. Doulgeraki, E. Karuzaki, I. Adami, S. Ntoa, D. Metilli, V. Bartalesi, C. Meghini, Y. Marketakis, D. Kaplanidi, M. Theodoridou, and X. Zabulis. 2021. Representation of socio-historical context to support the authoring and presentation of multimodal narratives: The Mingei Online Platform. fournal on Computing and Cultural Heritage (2021), 15, 1, Article 16 (February 2022), https://doi.org/10.1145/3465556

[70] N. Partarakis, X. Zabulis, A. Chatziantoniou, N. Patsiouras, and I. Adami. 2020. An approach to the creation and presentation of reference gesture datasets for the preservation of traditional crafts. Applied Sciences 10, 11 (2020), 7325. https://doi.org/10.3390/app10207325

[71] M. Peterson and E. Salvagio. 2010. Figure-ground perception. Scholarpedia 5, 4 (2010), 1-1. https://doi.org/10.4249/scholarpedia.4320 Revision 91261.

[72] I. Petri and F. Julien. 2017. Digitising the Performing Arts. Assessment report. Canadian Arts Presenting Association and Strategic Moves. https://capacoa.ca/en/research/digitizing-performing-arts/. Accessed on 2 May 2021.

[73] Mingei Online Platform. 2019. Baltadaki 2. http://mop.mingei-project.eu/resource/?uri=http://www.mingei-project.eu/resource/ 0e6824af-460c-4f47-a147-0527673bd6b8\&tab=summary. Tool. Accessed on 2 May 2021.

[74] Mingei Online Platform. 2019. The Massacre of Chios. http://mop.mingei-project.eu/resource/?uri=http://www.mingei-project.eu/ resource/147bcb9c-3427-4638-8cf1-13e0824357ea\&tab=summary. Event. Accessed on 2 May 2021.

[75] Mingei Online Platform. 2019. Pyrgi. http://mop.mingei-project.eu/resource/?uri=http://www.mingei-project.eu/resource/9dc66de09bf6-4b03-b385-ec5cc937ffb2\&tab=summary. Location. Accessed on 2 May 2021.

[76] Mingei Online Platform. 2019. Reconstruction of Pyrgi Village. http://mop.mingei-project.eu/resource/?uri=http://www.mingeiproject.eu/resource/052e24b6-a070-4cdb-970f-196f0ee5fbb4\&tab=summary. 3D model. Accessed on 2 May 2021.

[77] Mingei Online Platform. 2019. Reconstruction of xystiri. http://mop.mingei-project.eu/resource/?uri=http://www.mingei-project.eu/ resource/05ad387f-7d81-4705-9963-e01f8987ea05\&tab=summary. 3D model. Accessed on 2 May 2021.

[78] Mingei Online Platform. 2019. Xysta, Pyrgi-06. http://mop.mingei-project.eu/resource/?uri=http://www.mingei-project.eu/resource/ 3adb7864-8102-4012-9e75-260056b46251\&tab=summary. Photograph. Accessed on 2 May 2021.

[79] Mingei Online Platform. 2020. Carafe Making Process. http://mop.mingei-project.eu/resource/?uri=http://www.mingei-project.eu/ resource/f36bbd12-e884-421f-b9a3-850f7f992b97\&tab=summary. Process. Accessed on 2 May 2021.

[80] Mingei Online Platform. 2020. Glass Schema. http://mop.mingei-project.eu/resource/?uri=http\%3A\%2F\%2Fwww.mingei-project.eu\% 2Fresource\%2F51309a64-7f68-4e47-84e9-6be61170c92a. Process schema. Accessed on 2 May 2021.

[81] M. Polanyi. 1958. Personal Knowledge: Towards a Post-Critical Philosophy. University of Chicago Press, Chicago, IL.

[82] Mingei project. 2020. Gold Thread Preparation. https://youtu.be/dahgDvtSCdI. Video. Accessed on 2 May 2021.

[83] Mingei project. 2020. Interactive Graphic Novel on Glass Work. https://youtu.be/UeX3wwQW7WE. Video. Accessed on 2 May 2021.

[84] Mingei project. 2020. Mastic Cultivation. https://youtu.be/aB4c-1Ltitw. Video. Accessed on 2 May 2021. 
[85] Mingei project. 2020. Motion Capture of Weaver Using a Loom with a Treadle. https://youtu.be/BZj1LxRmByY. Video. Accessed on 2 May 2021.

[86] Mingei project. 2020. Psiloritis UNESCO Global Geopark (Greece). https://youtu.be/FHCJU7mkbOw. Video. Accessed on 2 May 2021.

[87] L. Pujol, M. Roussou, S. Poulou, O. Balet, M. Vayanou, and Y. Ioannidis. 2012. Personalizing interactive digital storytelling in archaeological museums: The CHESS project. In Annual Conference of Computer Applications and Quantitative Methods in Archaeology. Amsterdam University Press, Amsterdam, Netherlands, 93-100.

[88] D. Pye. 1968. The Nature and Art of Workmanship. Cambridge University Press, Cambridge, UK.

[89] A. Qammaz, D. Michel, and A. Argyros. 2018. A hybrid method for 3D pose estimation of personalized human body models. In IEEE Winter Conference on Applications of Computer Vision. IEEE Computer Society, 456-465. https://doi.org/10.1109/WACV.2018.00056

[90] A. Rigaki, N. Partarakis, and X. Zabulis. 2021. The Making of the Bontemps Carafe. https://doi.org/10.5281/zenodo.4750880

[91] A. Rigaki, N. Partarakis, X. Zabulis, and C. Stephanidis. 2020. An approach towards artistic visualizations of human motion in static media inspired by the visual arts. In International Conference on Advances in Computer-Human Interactions. International Academy, Research, and Industry Association, Valencia, Spain, 264-270. https://doi.org/10.5281/zenodo.4409665

[92] M. Ronkko and J. Lepisto. 2016. The craft process developing student decision making. Techne Series: Research in Sloyd Education and Craft Science A 23 (Jan. 2016), 48-61.

[93] C. Rother, V. Kolmogorov, and A. Blake. 2004. GrabCut interactive foreground extraction using iterated graph cuts. ACM Transactions on Graphics 23, 3 (Aug. 2004), 309-314.

[94] J. Sánchez, G. Mühlberger, B. Gatos, P. Schofield, K. Depuydt, R. Davis, E. Vidal, and J. de Does. 2013. TranScriptorium: A European project on handwritten text recognition. In ACM Symposium on Document Engineering (Florence, Italy). Association for Computing Machinery, New York, NY, 227-228. https://doi.org/10.1145/2494266.2494294

[95] N. Sarafianos, B. Boteanu, B. Ionescu, and I. Kakadiaris. 2016. 3D human pose estimation: A review of the literature and analysis of covariates. Computer Vision and Image Understanding 152 (2016), 1-20. https://doi.org/10.1016/j.cviu.2016.09.002

[96] G. Shi, Y. Wang, and S. Li. 2014. Human motion capture system and its sensor analysis. Sensors and Transducers 172, 6 (2014), 206-212.

[97] AG Software. 2010. Process Intelligence For Dummies. Wiley Publishing, Inc., Hoboken, New Jersey.

[98] C. Sporleder. 2010. Natural language processing for cultural heritage domains. Language and Linguistics Compass 4, 9 (2010), $750-768$. https://doi.org/10.1111/j.1749-818X.2010.00230.x

[99] E. Stefanidi, N. Partarakis, X. Zabulis, I. Adami, S. Ntoa, and G. Papagiannakis. 2021. Transferring traditional crafts from the physical to the virtual world: An authoring and visualization method and platform. ACM fournal of Computing and Cultural Heritage (2021), 15, 2, Article 35 (June 2022), 24 pages. https://doi.org/10.1145/3484397

[100] Y. Syu, Li-o Chen, and Yu-Fang Tu. 2018. A case study of digital preservation of motion capture for Bā Jiā Jiāng performance, Taiwan religious performing arts. In Digital Heritage. Progress in Cultural Heritage: Documentation, Preservation, and Protection. Springer International Publishing, Cham, 103-110.

[101] S. Tanaka. 2013. The notion of embodied knowledge and its range. Encyclopaideia: fournal of Phenomenology and Education 37 (2013), 47-66.

[102] UNESCO. 2003. Representative List of the Intangible Cultural Heritage of Humanity. https://ich.unesco.org/en/lists. Accessed on 2 May 2021.

[103] UNESCO. 2003. Text of the Convention for the Safeguarding of the Intangible Cultural Heritage.

[104] UNESCO. 2014. Mali Launches an Inventory of Intangible Cultural Heritage. https://ich.unesco.org/en/news/mali-launches-aninventory-of-intangible-cultural-heritage-00084. Press release. Accessed on 2 May 2021.

[105] UNSECO. 2014. Know-How of Cultivating Mastic on the Island of Chios. Inscription 00933 on Representative List of the Intangible Cultural Heritage of Humanity. https://ich.unesco.org/en/RL/know-how-of-cultivating-mastic-on-the-island-of-chios-00993. Accessed on 2 May 2021.

[106] UNSECO. 2015. Tinian Marble Craftsmanship. Inscription 01103 on Representative List of the Intangible Cultural Heritage of Humanity. https://ich.unesco.org/en/RL/tinian-marble-craftsmanship-01103. Accessed on 2 May 2021.

[107] UNSECO. 2018. Art of Dry Stone Walling, Knowledge and Techniques. Inscription 01393 on Representative List of the Intangible Cultural Heritage of Humanity. https://ich.unesco.org/en/RL/art-of-dry-stone-walling-knowledge-and-techniques-01393. Accessed on 2 May 2021.

[108] P. Vannini and A. Vannini. 2020. Artisanal ethnography: Notes on the making of ethnographic craft. Qualitative Inquiry 26, 7 (2020), 865-874. https://doi.org/10.1177/1077800419863456

[109] K. Wang, Y. Liao, W. Chu, J. Chiang, Y. Chen, and P. Chan. 2011. Digitization and value-add application of bamboo weaving artifacts. In International Conference on Asian Digital Libraries. Springer, 16-25.

[110] X. Zabulis, C. Meghini, N. Partarakis, D. Kaplanidi, P. Doulgeraki, E. Karuzaki, E. Stefanidi, T. Evdemon, D. Metilli, V. Bartalesi, M. Fasoula, E. Tasiopoulou, and C. Beisswenger. 2019. What is needed to digitise knowledge on heritage crafts? Memoriamedia Review 4 (2019), 1-25. https://doi.org/10.5281/zenodo.3723792

Received May 2021; revised October 2021; accepted October 2021 